\title{
DETECTION OF STRUCTURAL CHANGES THROUGH PRINCIPAL COMPONENT ANALYSIS AND MULTIVARIATE STATISTICAL INFERENCE
}

\author{
Francesc Pozo*, Ignacio Arruga ${ }^{\dagger}$, Luis E. Mujica*, Elena Podivilova ${ }^{\ddagger}$ \\ ${ }^{\star}$ CoDAlab (codalab.ma3. upc . edu), Departament de Matemàtica Aplicada III, Escola Universitària \\ d'Enginyeria Tècnica Industrial de Barcelona (EUETIB), Universitat Politècnica de Catalunya (UPC) \\ Comte d'Urgell, 187, E-08036 Barcelona, Spain \\ francesc.pozo@upc.edu, luis.eduardo.mujica@upc.edu \\ ${ }^{\dagger}$ Departamento de Seguridad Pasiva, IDIADA Automotive Technology SA \\ Polígon Industrial L'Albornar, s/n, 43710 Santa Oliva, Tarragona, Spain \\ nacho_arruga@hotmail.com \\ ${ }^{\ddagger}$ South Ural State University (National Research University), Control Systems Department \\ 454021, Lenin Prospekt, 76, Chelyabinsk, Russia \\ podivilovaeo@susu.ac.ru
}

Keywords: damage detection, PCA, multivariate hypothesis testing, structural health monitoring.

Summary: This paper is focused on the development of a damage detection indicator that combines a data driven baseline model (reference pattern obtained from the healthy structure) based on principal component analysis (PCA) and multivariate hypothesis testing. More precisely, a test for the plausibility of a value for a normal population mean vector is performed. The results indicate that the test is able to accurately clasify random samples as healthy or not.

\section{INTRODUCTION}

Among all the elements that integrate a structural health monitoring (SHM) system, methods or strategies for damage detection are nowadays playing a key role for improving the operational reliability of critical structures in several industrial sectors [2]. The essential paradigm is that a self-diagnosis and some level of detection and classification of damage is possible through the comparison of the in-service dynamic time responses of a structure with respect to baseline reference responses recorded in ideal healthy operating conditions [3]. These dynamic time responses recorded in each test, even in stable environmental and operational conditions, present the main characteristic that they are not repeatable. It means that always exist variation between measurements. Such variability may be caused by random measurement errors: measure instruments are often not perfectly calibrated and thus generating discordant interpretation and report of the results. 
Since the dynamic response of a structure can be considered as a random variable, a set of dynamic responses gathered from several experiments can be defined as a sample variable and, all possible values of the dynamic response as the population variable. Therefore, the process to draw conclusions about the state of the structure from several experiments by using statistical methods is usually named as statistical inference for damage diagnosis. In SHM field, statistical inference can be considered as one of the emerging technologies that will have an impact on the damage prognosis process $[4,5]$.

In general, there are two kinds of statistical inference: (i) estimation, which uses sample variables to predict an unknown parameter of the population variable and, (ii) hypothesis testing, which uses sample variables to determine whether a parameter fulfils a specific condition and to test a hypotheses about a population variable. In this last context, classical hypothesis test is used to compare extracted statistical quantities from statistical time series models like mean, normalized autocovariance function, cross covariance function, power spectral density, cross spectral density, frequency response function, squared coherence, residual variance, likelihood function, residual sequences, among others [7]. A hypothesis testing technique called a sequential probability ratio test (SPRT) has been combined with time series analysis and neural networks for damage classification in [18]. The usefulness of the proposed approach is demonstrated using a numerical example of a computer hard disk and an experimental study of an eight degree-of-freedom spring-mass system. Afterwards, the performance of the SPRT is improved by integrating extreme values statistics, which specifically models behavior in the tails of the distribution of interest into the SPRT. A three-story building model was constructed in a laboratory environment to assess the approach [19]. Recently, a generalized likelihood ratio test (GLRT) is used to compare the fit of minimum mean square error MMSE model parameters in order to detect damages in a scaled wooden model bridge [10, 11].

In previous works, the authors have been investigating novel multi-actuator piezoelectric systems for detection and localization of damages. These approaches combine: (i) the dynamic response of the structure at different exciting and receiving points; (ii) the correlation of dynamical responses when some damage appear in the structure by using principal component analysis (PCA) and statistical measures that are used as damage indices; and (iii) the contribution of each sensor to the indices, what is used to localize the damage [14, 13].

Following the same framework and considering dynamic responses as random variables as in [15], this paper is focused on the development of a damage detection indicator that combines a data driven baseline model (reference pattern obtained from the healthy structure) based on principal component analysis (PCA) and multivariate hypothesis testing. As said before, the use of hypothesis testing is not new in this field. The novelty of the previous work [15] is based on (i) the nature of the data used in the test since we are using scores instead of the measured response of the structure [7] or the coefficients of an AutoRegressive model [21]; (ii) the number of data used since our test is based on two random samples instead of two characteristic quantities [6]. The proposed development starts obtaining the baseline PCA model and the subsequent projections using the healthy structure. When the structure needs to be inspected, new experiments are performed and they are projected onto the baseline PCA model. Each exper- 
iment is considered as a random process and the projections onto a predetermined number of principal component is a multivariate random variable. The objective is to analyse whether the distribution of the variable associated with the current structure is related to the healthy one.

\section{DATA DRIVEN BASELINE MODEL BASED ON PCA}

In this work a particular experimental set-up based on the analysis of vibrational changes is used as an exemplifying configuration in order to justify, validate and test the methodology. The proposed methodology can also be applied to a more general structure.

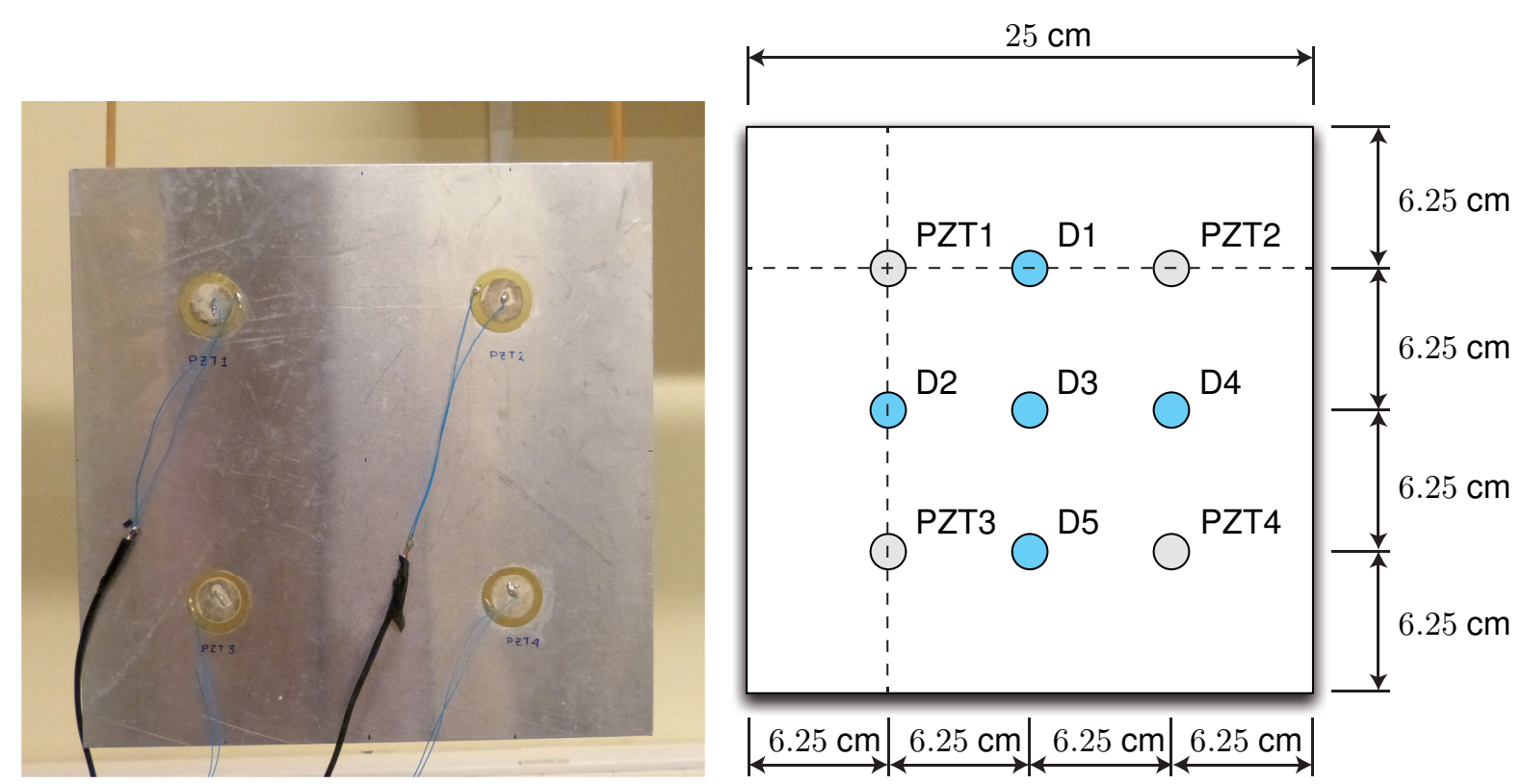

Figure 1. Aluminium plate (left). Dimensions and piezoelectric transducers location (right).

\subsection{Experimental set-up}

Some experiments were performed in order to test the methods presented on this paper. In these experiments, four piezoelectric transducer discs (PZTs) were attached to the surface of a thin aluminum plate, with dimensions $250 \mathrm{~mm} \times 250 \mathrm{~mm} \times 1 \mathrm{~mm}$. Those PZTs formed a square with $144 \mathrm{~mm}$ per side. The plate was suspended by two elastic ropes, being isolated from environmental influences. Figure 1 (left) shows the plate hanging on the elastic ropes.

As a response to an electrical excitation, a PZT produces a mechanical vibration, propagating, in this case, across the plate (forming Lamb waves, since a thin plate has been used). PZTs are also able to generate an electrical signal as a response to a mechanical vibration. In every excitation phase of an experiment, one PZT were used as actuator and the other three PZTs were used as sensors, recording the dynamical response of the plate.

500 experiments were performed over the healthy structure, and another 500 experiments 
were performed over the damaged structure with 5 damage types (100 experiments per damage type). Figure 1 (right) shows the position of damages 1 to 5 (D1 to D5). As excitation, a $50 \mathrm{kHz}$ sinusoidal signal modulated by a hamming window were used. Figure 3 shows the excitation signal and an example of the signal collected by PZT 1.

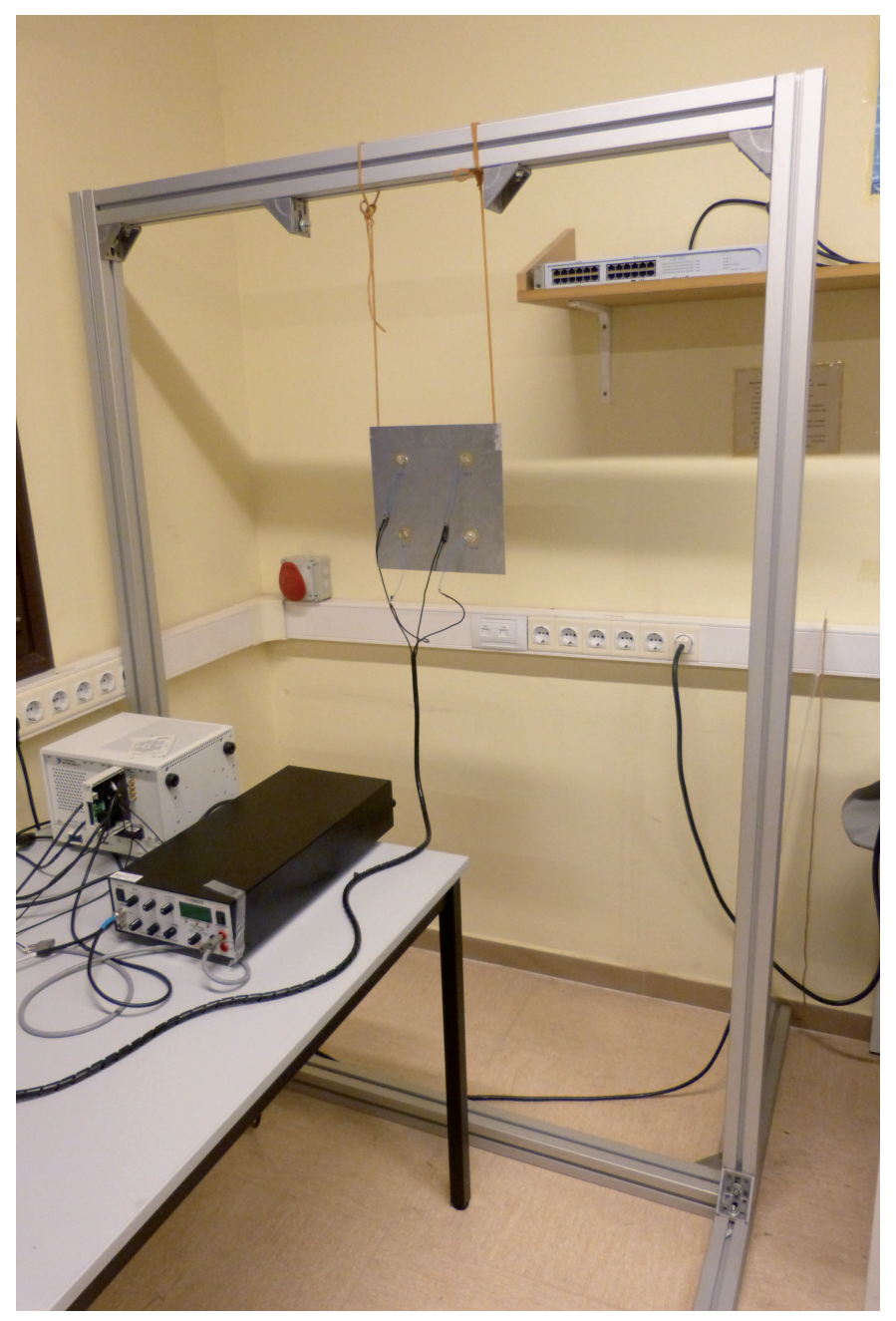

Figure 2. The plate is suspended by two elastic ropes in a metallic frame.

\subsection{Principal component analysis (PCA): theoretical background}

Let us initiate the analysis of a physical process by measuring different variables (sensors) at a finite number of time instants. In this work, the collected data are arranged in a $n \times(N \cdot L)$ 
matrix as follows:

$$
\mathbf{X}=\left(\begin{array}{ccccccccccc}
x_{11}^{1} & x_{12}^{1} & \cdots & x_{1 L}^{1} & x_{11}^{2} & \cdots & x_{1 L}^{2} & \cdots & x_{11}^{N} & \cdots & x_{1 L}^{N} \\
\vdots & \vdots & \ddots & \vdots & \vdots & \ddots & \vdots & \ddots & \vdots & \ddots & \vdots \\
x_{i 1}^{1} & x_{i 2}^{1} & \cdots & x_{i L}^{1} & x_{i 1}^{2} & \cdots & x_{i L}^{2} & \cdots & x_{i 1}^{N} & \cdots & x_{i L}^{N} \\
\vdots & \vdots & \ddots & \vdots & \vdots & \ddots & \vdots & \ddots & \vdots & \ddots & \vdots \\
x_{n 1}^{1} & x_{n 2}^{1} & \cdots & x_{n L}^{1} & x_{n 1}^{2} & \cdots & x_{n L}^{2} & \cdots & x_{n 1}^{N} & \cdots & x_{n L}^{N}
\end{array}\right)
$$

Matrix $\mathbf{X} \in \mathcal{M}_{n \times(N \cdot L)}(\mathbb{R})$-where $\mathcal{M}_{n \times(N \cdot L)}(\mathbb{R})$ is the vector space of $n \times(N \cdot L)$ matrices over $\mathbb{R}$ - contains data from $N$ sensors at $L$ discretization instants with respect to $n$ experimental trials. Consequently, each row vector $\mathbf{X}(i,:) \in \mathbb{R}^{N \cdot L}, i=1, \ldots, n$, represents, for a specific experimental trial, the measurements from all the sensors at every specific time instant. Equivalently, each column vector $\mathbf{X}(:, j) \in \mathbb{R}^{n}, j=1, \ldots, N \cdot L$, represents measurements from one sensor at one particular time instant in the whole set of experimental trials.
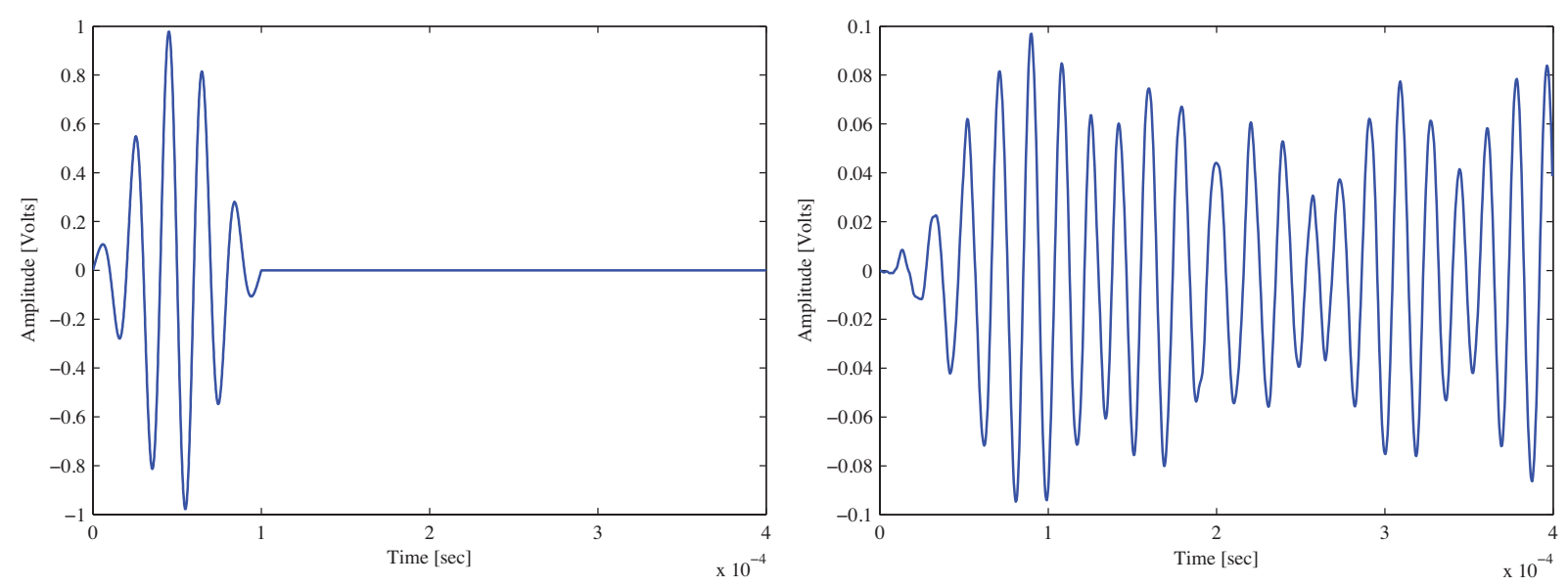

Figure 3. Excitation signal (above) and, dynamic response recorded by PZT 1 (below).

The main objective of principal component analysis (PCA) is to distinguish which dynamics are more relevant in the system, which are redundant and which can be considered as a noise [14]. This objective is essentially accomplished by defining a new coordinate space to reexpress the original one. This new coordinate space is used to filter noise and redundancies according to the variance-covariance matrix of the original data. In other words, the objective is to find a linear transformation orthogonal matrix $\mathbf{P} \in \mathcal{M}_{(N \cdot L) \times(N \cdot L)}(\mathbb{R})$ that will be used to transform the original data matrix $\mathrm{X}$ according to the following matrix multiplication

$$
\mathbf{T}=\mathbf{X P} \in \mathcal{M}_{n \times(N \cdot L)}(\mathbb{R}) .
$$

Matrix $\mathbf{P}$ is usually called the principal components of the data set or loading matrix and matrix $\mathbf{T}$ is the transformed or projected matrix to the principal component space, also called score 
matrix. Using all the $N \cdot L$ principal components, that is, in the full dimensional case, the orthogonality of $\mathbf{P}$ implies $\mathbf{P} \mathbf{P}^{\mathrm{T}}=\mathbf{I}$, where $\mathbf{I}$ is the $(N \cdot L) \times(N \cdot L)$ identity matrix. Therefore, the projection can be inverted to recover the original data as

$$
\mathbf{X}=\mathbf{T P}^{T} \text {. }
$$

Matrix $\mathbf{P}$ can be computed by means of the singular value decomposition (SVD) of the covariance matrix defined in equation (3). Then, the principal components are defined by the eigenvectors and eigenvalues of the covariance matrix as follows:

$$
\begin{aligned}
\mathbf{C}_{\mathbf{X}} & =\frac{1}{N \cdot L-1} \mathbf{X}^{T} \mathbf{X} \in \mathcal{M}_{(N \cdot L) \times(N \cdot L)}(\mathbb{R}), \\
\mathbf{C}_{\mathbf{X}} \mathbf{P} & =\mathbf{P} \Lambda,
\end{aligned}
$$

where the columns of $\mathbf{P}$ are the eigenvectors of $\mathbf{C}_{\mathbf{X}}$. The diagonal terms of matrix $\Lambda$ are the eigenvalues $\lambda_{i}, i=1, \ldots, N \cdot L$, of $\mathbf{C}_{\mathbf{X}}$ whereas the off-diagonal terms are zero, that is,

$$
\begin{aligned}
& \Lambda_{i i}=\lambda_{i}, i=1, \ldots, N \cdot L \\
& \Lambda_{i j}=0, i, j=1, \ldots, N \cdot L, i \neq j
\end{aligned}
$$

The eigenvectors $p_{j}, j=1, \ldots, N \cdot L$, representing the columns of the transformation matrix $\mathbf{P}$ are classified according to the eigenvalues in descending order and they are called the principal components of the data set. The eigenvector with the highest eigenvalue, called the first principal component, represents the most important pattern in the data with the largest quantity of information.

However, the objective of PCA is, as said before, to reduce the dimensionality of the data set $\mathrm{X}$ by selecting only a limited number $\ell<N \cdot L$ of principal components, that is, only the eigenvectors related to the $\ell$ highest eigenvalues. Thus, given the reduced matrix

$$
\hat{\mathbf{P}}=\left(p_{1}\left|p_{2}\right| \cdots \mid p_{\ell}\right) \in \mathcal{M}_{(N \cdot L) \times \ell}(\mathbb{R}),
$$

matrix $\hat{\mathbf{T}}$ is defined as

$$
\hat{\mathbf{T}}=\mathbf{X} \hat{\mathbf{P}} \in \mathcal{M}_{n \times \ell}(\mathbb{R}) .
$$

Note that opposite to $\mathbf{T}, \hat{\mathbf{T}}$ is no longer invertible. Consequently, it is not possible to fully recover $\mathbf{X}$ although $\hat{\mathbf{T}}$ can be projected back onto the original $(N \cdot L)$-dimensional space to get a data matrix $\hat{\mathbf{X}}$ as follows:

$$
\hat{\mathbf{X}}=\hat{\mathbf{T}} \hat{\mathbf{P}}^{T} \in \mathcal{M}_{n \times(N \cdot L)}(\mathbb{R}) .
$$

The difference between the original data matrix $\mathbf{X}$ and $\hat{\mathbf{X}}$ is defined as the residual error matrix $\mathbf{E}$ as follows:

$$
\mathbf{E}=\mathbf{X}-\hat{\mathbf{X}}
$$


or, equivalenty,

$$
\mathbf{X}=\hat{\mathbf{X}}+\mathbf{E}=\hat{\mathbf{T}} \hat{\mathbf{P}}^{T}+\mathbf{E}
$$

The residual error matrix $\mathbf{E}$ describes the variability not represented by the data matrix $\hat{\mathbf{X}}$.

Even though the real measures obtained from the sensors as a function of time represent physical magnitudes, when these measures are projected and the scores are obtained, these scores no longer represent any physical magnitude [15]. The key point in this approach is that the scores from different experiments can be compared with the reference pattern to try to detect a contrasting behavior.

\subsection{PCA modelling}

For the PCA modelling stage, we carry out a set of experiments as stated in Section 2.1 . For each different phase (PZT1 will act as an actuator in phase 1, PZT2 will act as an actuator in phase 2 and so on) and considering the signals measured by the sensors, the matrix $\mathbf{X}_{\mathrm{h}}$ is defined and arranged as in equation (1) and scaled as stated in [14]. PCA modelling basically consists of computing the projection matrix $\mathbf{P}$ for each phase as in equation (2). Matrix $\mathbf{P}$, renamed $\mathbf{P}_{\text {model }}$, provides an improved and dimensionally limited representation of the original data $\mathbf{X}_{\mathrm{h}} . \mathbf{P}_{\text {model }}$ is considered as the model of the healthy structure to be used to detect structural damage. The modelling stage is graphically represented in Figure 4.

\section{DETECTION OF STRUCTURAL CHANGES BASED ON MULTIVARIATE STA- TISTICAL INFERENCE}

A predetermined number of experiments is performed in the structure to be diagnosed and a new data matrix $\mathbf{X}_{\mathrm{c}}$ is constructed with the recorded data, as in equation (1). The number of experiments is not limited a priori. However, the number of sensors and recorded samples must correspond with the number of sensors and recorded samples in the PCA modelling stage; more precisely, the number of columns of $\mathbf{X}_{\mathrm{c}}$ and $\mathbf{X}_{\mathrm{h}}$ must agree. Matrix $\mathbf{X}_{\mathrm{c}}$ will be projected onto the PCA model as specified in Section 3.1 The projections onto the first components -the socalled scores - are used for the construction of the multivariate random samples to be compared and consequently to obtain the structural damage indicator, as it is illustrated in Figure 5.

\subsection{Multivariate random variables and multivariate random samples}

Let us start this section by specifying what we consider a random variable and how a multivariate random variable is built. Assume that for a specific actuator phase (for instance, PZT $i$ as actuator, $i=1,2,3,4)$ and using the signals measured by the sensors in a fully healthy state the baseline PCA model (identified as $\mathbf{P}_{\text {model }}^{i}$ ) is built as in Sections 2.2and 2.3 Assume also that an experiment as detailed in section 2. lis further performed. The time responses recorded by the sensors are first discretized and then arranged in a row vector $r^{i} \in \mathbb{R}^{N \cdot L}$, where $N$ is the number of sensors, $L$ is the number of discretization instants and $i$ refers to the current actuator 


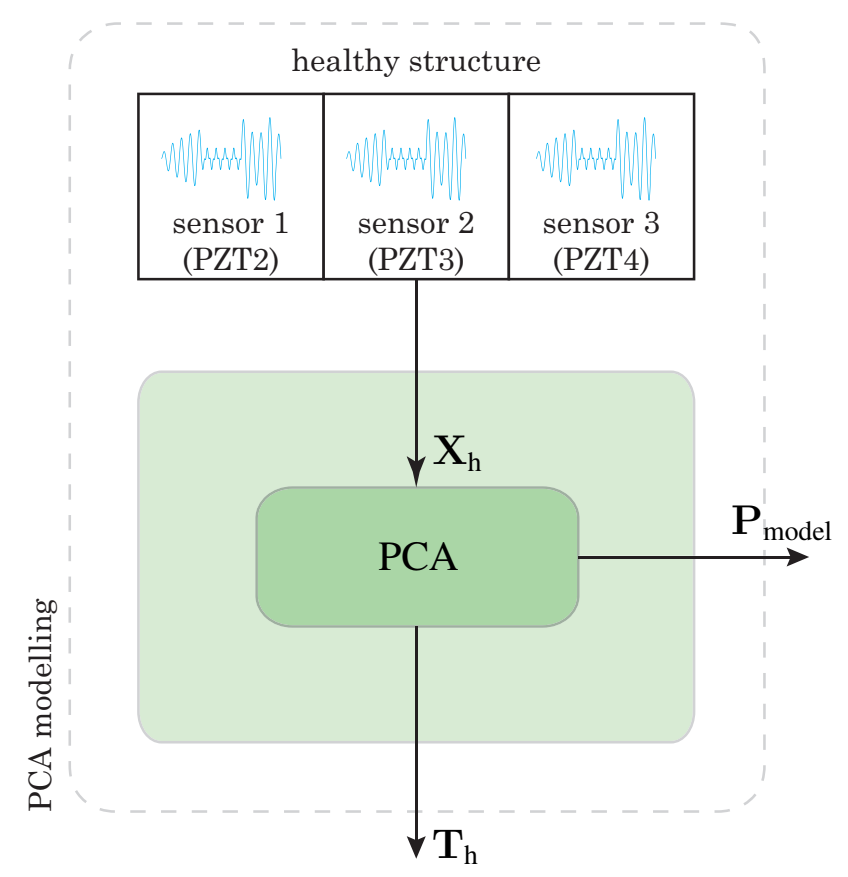

Figure 4. A PCA model $\mathbf{P}_{\text {model }}$ is built for each actuator phase using the signals $\mathbf{X}_{\mathrm{h}}$ recorded by sensors during the experiments with the undamaged structure.

phase. The number of sensors and discretization instants must be equal to those that were used when defining $\mathbf{P}_{\text {model }}^{i}$. Besides, the size of each column is $N \cdot L$. Selecting the $j$ th principal component $(j=1, \ldots, \ell)$,

$$
\mathbf{P}_{\text {model }}^{i}(:, j)=: v_{j}^{i} \in \mathbb{R}^{N \cdot L},
$$

the projection of the recorded data onto this principal component is the dot product

$$
t_{j}^{i}=r^{i} \cdot v_{j}^{i} \in \mathbb{R}
$$

as in equation (2).

Since the dynamic behaviour of a structure depends on some indeterminacy, its dynamic response can be considered as a stochastic process and the measurements in $r^{i}$ are also stochastic. On the one hand, $t_{j}^{i}$ acquires this stochastic nature and it will be regarded as a random variable to construct the stochastic approach in this paper. On the other hand, an $s$-dimensional random vector can be defined by considering the projections onto several principal components as follows

$$
\mathbf{t}_{j_{1}, \ldots, j_{s}}^{i}=\left[\begin{array}{llll}
t_{j_{1}}^{i} & t_{j_{2}}^{i} & \cdots & t_{j_{s}}^{i}
\end{array}\right]^{T} \in \mathbb{R}^{s}, s \in \mathbb{N}, j_{1}, \ldots, j_{s} \in\{1, \ldots, \ell\}, j_{\alpha} \neq j_{\beta} \text { if } \alpha \neq \beta .
$$




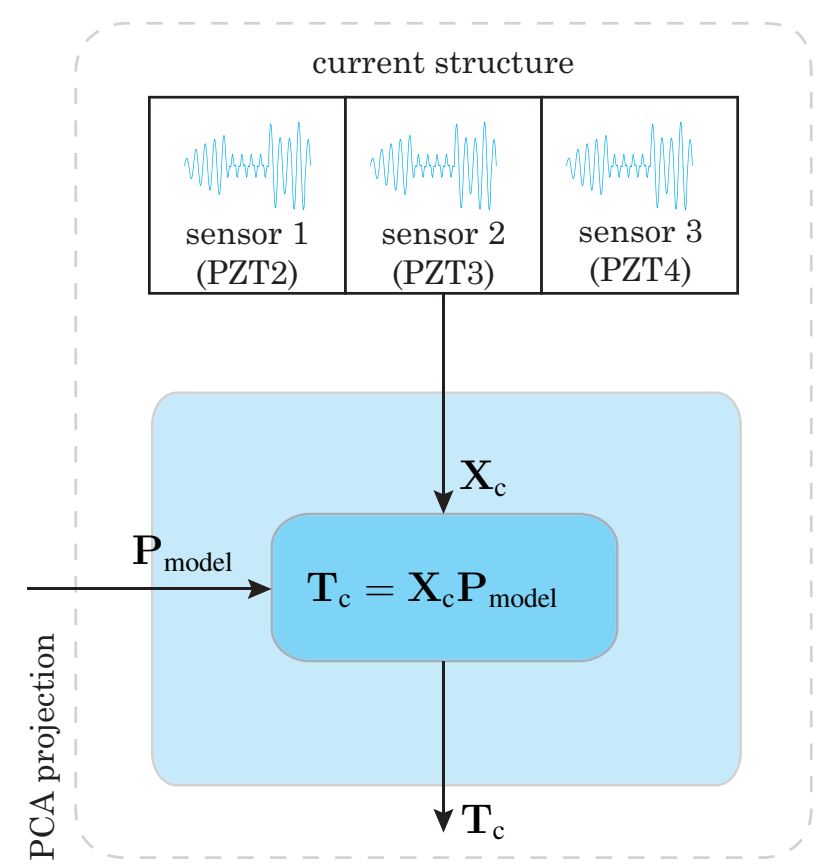

Figure 5. The structure to be diagnosed is subjected to a predefined number of experiments and a data matrix $\mathbf{X}_{c}$ is constructed. This matrix is projected onto the baseline PCA model $\mathbf{P}_{\text {model }}$ to obtain the projections onto the first components $\mathbf{T}_{\mathrm{c}}$.

By reiterating this experiment several times on the undamaged structure and using equations (8)-(9) we have a multivariate random sample of the variable $\mathbf{t}_{j_{1}, \ldots, j_{s}}^{i}$ that can be viewed as a baseline. When structural changes on the structure have to be detected, a new set of experiments should be performed to create the multivariate random sample that will be compared with the multivariate baseline sample. As an example, in Figure 6 two three-dimensional samples are represented; one is the three-dimensional baseline sample (left) and the other is referred to damages 1 to 3 (right). This illustrating example refers to actuator phase 1 and the first, second and third principal components. More precisely, Figure 6 (right) depicts the values of the multivariate random variable $\mathbf{t}_{1,2,3}^{1}$. The diagnosis sample is formed by 20 experiments and the baseline sample is made by 100 experiments.

\subsection{Detection phase}

In this work, the framework of multivariate statistical inference is used with the objective of the classification of structures in healthy or damaged. With this goal, a test for multivariate normality is first performed. A test for the plausibility of a value for a normal population mean vector is then performed. 

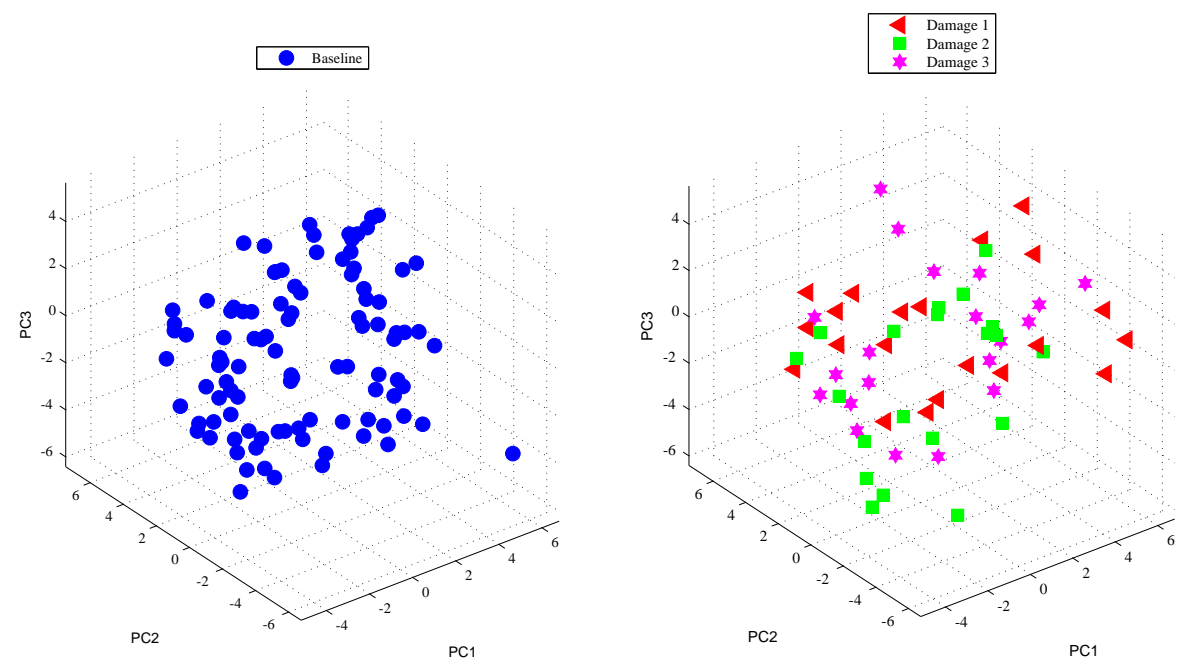

Figure 6. Baseline sample (left) and sample from the structure to be diagnosed (right).

\subsubsection{Testing for multivariate normality}

Many statistical tests and graphical approaches are available to check the multivariate normality assumption [16]. But there is no a single most powerful test and it is recommended to perform several tests to assess the multivariate normality. Let us consider the three most widely used multivariate normality tests. That is: (i) Mardia's; (ii) Henze-Zirkler's; and (iii) Royston's multivariate normality tests. We include a brief explanation of these methods for the sake of completeness.

Mardia's test is based on multivariate extensions of skewness $\left(\hat{\gamma}_{1, s}\right)$ and kurtosis $\left(\hat{\gamma}_{2, s}\right)$ measures $[9,16]$

$$
\begin{aligned}
& \hat{\gamma}_{1, s}=\frac{1}{\nu^{2}} \sum_{i=1}^{\nu} \sum_{j=1}^{\nu} m_{i j}^{3}, \\
& \hat{\gamma}_{2, s}=\frac{1}{\nu} \sum_{i=1}^{\nu} m_{i j}^{2}
\end{aligned}
$$

where $m_{i j}=\left(x_{i}-\bar{x}\right)^{T} S^{-1}\left(x_{j}-\bar{x}\right), i, j=1, \ldots, \nu$ is the squared Mahalanobis distance, $S$ is the variance-covariance matrix, $s$ is the number of variables and $\nu$ is the sample size. The test statistic for skewness, $(\nu / 6) \hat{\gamma}_{1, s}$, is approximately $\chi^{2}$ distributed with $s(s+1)(s+2) / 6$ degrees of freedom. Similarly, the test statistic for kurtosis, $\hat{\gamma}_{2, s}$, is approximately normally distributed with mean $s(s+2)$ and variance $8 s(s+2) / \nu$. For multivariate normality, both $p$-values of skewness and kurtosis statistics should be greater than 0.05 .

For small samples, the power and the type I error could be violated. Therefore, Mardia introduced a correction term into the skewness test statistic [12], usually when $\nu<20$, in order 
to control type I errors. The corrected skewness statistic for small samples is $(\nu k / 6) \hat{\gamma}_{1, s}$, where $k=(s+1)(\nu+1)(\nu+3) /(\nu(\nu+1)(s+1)-6)$. This statistic is also $\chi^{2}$ distributed with $s(s+1)(s+2) / 6$ degrees of freedom.

The Henze-Zirkler's test is based on a non-negative functional distance that measures the distance between two distribution functions $[8,9]$. If the data is multivariate normal distributed, the test statistic $H Z$ in equation (10) is approximately lognormally distributed. It proceeds to calculate the mean, variance and smoothness parameter. Then, mean and variance are lognormalized and the $p$-value is estimated. The test statistic of Henze-Zirkler's multivariate normality test is

$$
H Z=\frac{1}{\nu} \sum_{i=1}^{\nu} \sum_{j=1}^{\nu} e^{-\frac{\beta^{2}}{2} D_{i j}}-2\left(1+\beta^{2}\right)^{-\frac{s}{2}} \sum_{i=1}^{\nu} e^{-\frac{\beta^{2}}{2\left(1+\beta^{2}\right)} D_{i}}+\nu\left(1+\beta^{2}\right)^{-\frac{s}{2}}
$$

where $s$ is the number of variables, $\beta=\frac{1}{\sqrt{2}}\left(\frac{\nu(2 s+1)}{4}\right)^{\frac{1}{s+4}}, D_{i j}=\left(x_{i}-x_{j}\right)^{T} S^{-1}\left(x_{i}-x_{j}\right), i, j=$ $1, \ldots, \nu$ and $D_{i}=\left(x_{i}-\bar{x}\right)^{T} S^{-1}\left(x_{i}-\bar{x}\right)=m_{i i}, i=1, \ldots, \nu$.

$D_{i}$ gives the squared Mahalanobis distance of the $i$ th observation to the centroid and $D_{i j}$ gives the Mahalanobis distance between the $i$ th and the $j$ th observations. If data are multivariate normal distributed, the test statistic $(H Z)$ is approximately lognormally distributed with mean $\mu$ and variance $\sigma^{2}$ as given below:

$$
\begin{aligned}
\mu & =1-\frac{a^{-\frac{s}{2}}\left(1+s \beta^{\frac{2}{a}}+s(s+2) \beta^{4}\right)}{2 a^{2}} \\
\sigma^{2} & =2\left(1+4 \beta^{2}\right)^{-\frac{s}{2}}+\frac{2 a^{-s}\left(1+2 s \beta^{4}\right)}{a^{2}}+\frac{3 s(s+2) \beta^{8}}{4 a^{4}}-4 w_{\beta}^{-\frac{s}{2}}\left(1+\frac{3 s \beta^{4}}{2 w_{\beta}}+\frac{s(s+2) \beta^{8}}{2 w_{\beta}^{2}}\right),
\end{aligned}
$$

where $a=1+2 \beta^{2}$ and $w_{\beta}=\left(1+\beta^{2}\right)\left(a+3 \beta^{2}\right)$. Hence, the lognormalized mean and variance of the $H Z$ statistic can be defined as follows:

$$
\begin{aligned}
& \mu_{\log }=\ln \left(\sqrt{\frac{\mu^{4}}{\sigma^{2}+\mu^{2}}}\right), \\
& \sigma_{\log }^{2}=\ln \left(\frac{\sigma^{2}+\mu^{2}}{\sigma^{2}}\right) .
\end{aligned}
$$

By using the lognormal distribution parameters, $\mu_{\log }$ and $\sigma_{\log }^{2}$, we can test the significance of multivariate normality. The Wald test statistic for multivariate normality is given in the following equation:

$$
z=\frac{\ln (H Z)-\mu_{\log }}{\sqrt{\sigma_{\log }^{2}}}
$$


Royston's test uses the Shapiro-Wilk/Shapiro-Francia statistic to test multivariate normality [9]. If kurtosis of the data is greater than 3, then it uses the Shapiro-Francia test for leptokurtic distributions. Otherwise, it uses the Shapiro-Wilk test for platykurtic distributions. The ShapiroWilk test statistic is:

$$
W=\frac{\left(\sum_{i=1}^{\nu}\left(a_{i} \cdot x_{(i)}\right)\right)^{2}}{\sum_{i=1}^{\nu}\left(x_{i}-\mu\right)^{2}},
$$

where $x_{(i)}$ is the $i$ th order statistic, that is, the $i$ th-smallest number in the sample, $\mu$ is the mean, $a=\frac{\mathbf{m}^{T} V^{-1}}{\sqrt{\mathbf{m}^{T} V^{-1} V^{-1} \mathbf{m}}}, V$ is the covariance matrix of the order statistics of a sample of $s$ standard normal random variables with expectation vector $\mathbf{m}$. Let $W_{j}$ be the Shapiro-Wilk/ShapiroFrancia test statistic for the $j$ th variable, $j=1, \ldots, s$, and $Z_{j}$ be the values obtained from the normality transformation proposed by [17]:

$$
\begin{aligned}
& \text { if } 4 \leq \nu \leq 11 \text { then } x=\nu \text { and } w_{j}=-\ln \left(\gamma-\ln \left(1-W_{j}\right)\right) \\
& \text { if } 12 \leq \nu \leq 2000 \text { then } x=\ln (\nu) \text { and } w_{j}=\ln \left(1-W_{j}\right) .
\end{aligned}
$$

Then transformed values of each random variable can be obtained from the following equation:

$$
Z_{j}=\frac{w_{j}-\mu}{\sigma}
$$

where $\gamma, \mu$ and $\sigma$ are derived from the polynomial approximations given in equations [17]:

$$
\begin{aligned}
\text { if } \quad 4 \leq \nu \leq 11 \quad \gamma & =-2.273+0.459 x, \\
\mu & =0.544-0.39978 x+0.025054 x^{2}-0.0006714 x^{3}, \\
\ln (\sigma) & =1.3822-0.77857 x+0.062767 x^{2}-0.0020322 x^{3}, \\
\text { if } \quad 12 \leq \nu \leq 2000 \quad \mu & =-1.5861-0.31082 x-0.083751 x^{2}+0.0038915 x^{3}, \\
\ln (\sigma) & =-0.4803-0.082676 x+0.0030302 x^{2} .
\end{aligned}
$$

The Royston's test statistic for multivariate normality as follows:

$$
H=\frac{\varepsilon \sum_{j=1}^{s} \psi_{j}}{s} \sim \chi_{\varepsilon}^{2}
$$

where $\varepsilon$ is the equivalent degrees of freedom (edf) and $\Phi(\cdot)$ is the cumulative distribution function for standard normal distribution such that,

$$
\begin{aligned}
\varepsilon & =s /(1+(s-1) \bar{c}), \\
\psi_{j} & =\left(\Phi^{-1}\left(\Phi\left(-Z_{j}\right) / 2\right)\right)^{2}, \quad j=1,2, \ldots, s .
\end{aligned}
$$

Another extra term $\bar{c}$ has to be calculated in order to continue with the statistical significance of Royston's test statistic. Let $R$ be the correlation matrix and $r_{i j}$ be the correlation between $i$ th 
and $j$ th variables. Then, the extra term can be found by using equation:

$$
\bar{c}=\sum_{i=1}^{s} \sum_{j \neq i} \frac{c_{i j}}{s(s-1)},
$$

where

$$
c_{i j}=g\left(r_{i j}, \nu\right)
$$

with the boundaries of $g(\cdot)$ as $g(0, \nu)=0$ and $g(1, \nu)=1$. The function $g(\cdot)$ is defined as follows:

$$
g(r, \nu)=r^{\lambda}\left(1-\frac{\mu}{\xi(\nu)}(1-r)^{\mu}\right) .
$$

The unknown parameters $\mu, \lambda$ and $\xi$ were estimated from a simulation study conducted by [17]. He found $\mu=0.715$ and $\lambda=5$ for sample size $10 \leq \nu \leq 2000$ and $\xi$ is a cubic function which can be obtained as follows:

$$
\xi(\nu)=0.21364+0.015124 \ln ^{2}(\nu)-0.0018034 \ln ^{3}(\nu)
$$

The described tests were performed for all the data. We summarize in Table 1 the results of the multivariate normality test when considering the first three principal components (PC1-PC3) for all the actuator phases.

Apart from the multivariate normality tests, some visual representations can also be used to test for multivariate normality. The quantile-quantile (Q-Q) plot is a widely used graphical approach to evaluate the agreement between two probability distributions $[9,16]$. Each axis refers to the quantiles of probability distributions to be compared, where one of the axes indicates theoretical quantiles (hypothesized quantiles) and the other indicates the observed quantiles. If the observed data fit hypothesized distribution, the points in the Q-Q plot will approximately lie on the bisectrix $y=x$. The sample quantiles for the Q-Q plot are obtained as follows. First we rank the observations $y_{1}, y_{2}, \ldots, y_{\nu}$ and denote the ordered values by $y_{(1)}, y_{(2)}, \ldots, y_{(\nu)}$; thus $y_{(1)} \leq y_{(2)} \leq \cdot \leq y_{(\nu)}$. Then the point $y_{(i)}$ is the $i / \nu$ sample quantile. The fraction $i / \nu$ is often changed to $(i-0.5) / \nu$ as a continuity correction. With this convention, $y_{(i)}$ is designated as the $(i-0.5) / \nu$ sample quantile. The population quantiles for the Q-Q plot are similarly defined corresponding to $(i-0.5) / \nu$. If we denote these by $q_{1}, q_{2}, \ldots, q_{\nu}$, then $q_{i}$ is the value below which a proportion $(i-0.5) / \nu$ of the observations in the population lie; that is, $(i-0.5) / \nu$ is the probability of getting an observation less than or equal to $q_{i}$. Formally, $q_{i}$ can be found for the standard normal random variable $Y$ with distribution $N(0,1)$ by solving

$$
\Phi\left(q_{i}\right)=P\left(Y<q_{i}\right)=\frac{i-0.5}{\nu}
$$

which would require numerical integration or tables of the cumulative standard normal distribution, $\Phi(x)$. Another benefit of using $(i-0.5) / \nu$ instead of $i / \nu$ is that $\nu / \nu=1$ would make 
Table 1. Results of the multivariate normality tests when considering the first three principal components (PC1-PC3) in the four actuator phases. "-" means that all the tests rejected multivariate normality, "+" means that at least one test indicated multivariate normality while the subindex shows the tests that indicated normality: 1 (Mardia's test), 2 (Henze-Zirkler's test) or 3 (Royston's test).

\begin{tabular}{rccrr}
\hline & PZT1 act. & PZT2 act. & PZT3 act. & PZT4 act. \\
\hline Undamaged (baseline) & - & $+_{2}$ & $+_{2}$ & - \\
Undamaged (first set to test) & - & $+_{1,2,3}$ & $+_{2}$ & - \\
Undamaged (second set to test) & $+_{1}$ & $+_{1,2}$ & - & - \\
Undamaged (third set to test) & - & $+_{2}$ & - & $+_{2,3}$ \\
Undamaged (fourth set to test) & - & - & - & $+_{1,2,3}$ \\
Undamaged (fifth set to test) & - & - & $+_{1}$ & $+_{1,3}$ \\
D1 & $+_{1,2,3}$ & $+_{2}$ & $+_{1,2}$ & $+_{3}$ \\
D2 & $+_{1,2,3}$ & $+_{1,2,3}$ & $+_{1}$ & $+_{1,3}$ \\
D3 & $+_{1,2,3}$ & $+_{2}$ & $+_{1,2}$ & - \\
D4 & $+_{2}$ & $+_{2,3}$ & - & $+_{3}$ \\
D5 & $+_{1,2,3}$ & - & $+_{1}$ & - \\
\hline
\end{tabular}

$q_{\nu}=+\infty$. The population need not have the same mean and variance as the sample, since changes in mean and variance merely change the slope and intercept of the plotted lie in the Q-Q plot. Therefore, we use the standard normal distribution, and the $q_{i}$ values can easily be found from a table of cumulative standard normal probabilities. We then plot the pairs $\left(q_{i}, y_{(i)}\right)$ and examine the resulting Q-Q plot for linearity. Some examples of Q-Q plots for the data we consider in this paper are shown on Figure 7. It can be observed that the points are distributed closely following the bisectrix, thus indicating the multivariate normality of the data as stated in Table 1.

In addition to Q-Q plots, creating perspective and contour plots can be also useful $[9,16]$. The perspective plot is an extension of the univariate probability distribution curve into a threedimensional probability distribution surface related with bivariate distributions. It also gives information about where data are gathered and how two variables are correlated with each other. It consists of three dimensions where two dimensions refer to the values of the two variables and the third dimension, which is likely in univariate cases, is the value of the multivariate normal probability density function. Another alternative graph, which is called the contour plot, involves the projection of the perspective plot into a two-dimensional space and this can be used for checking multivariate normality assumption. Figure 8 shows the contour plot for bivariate 

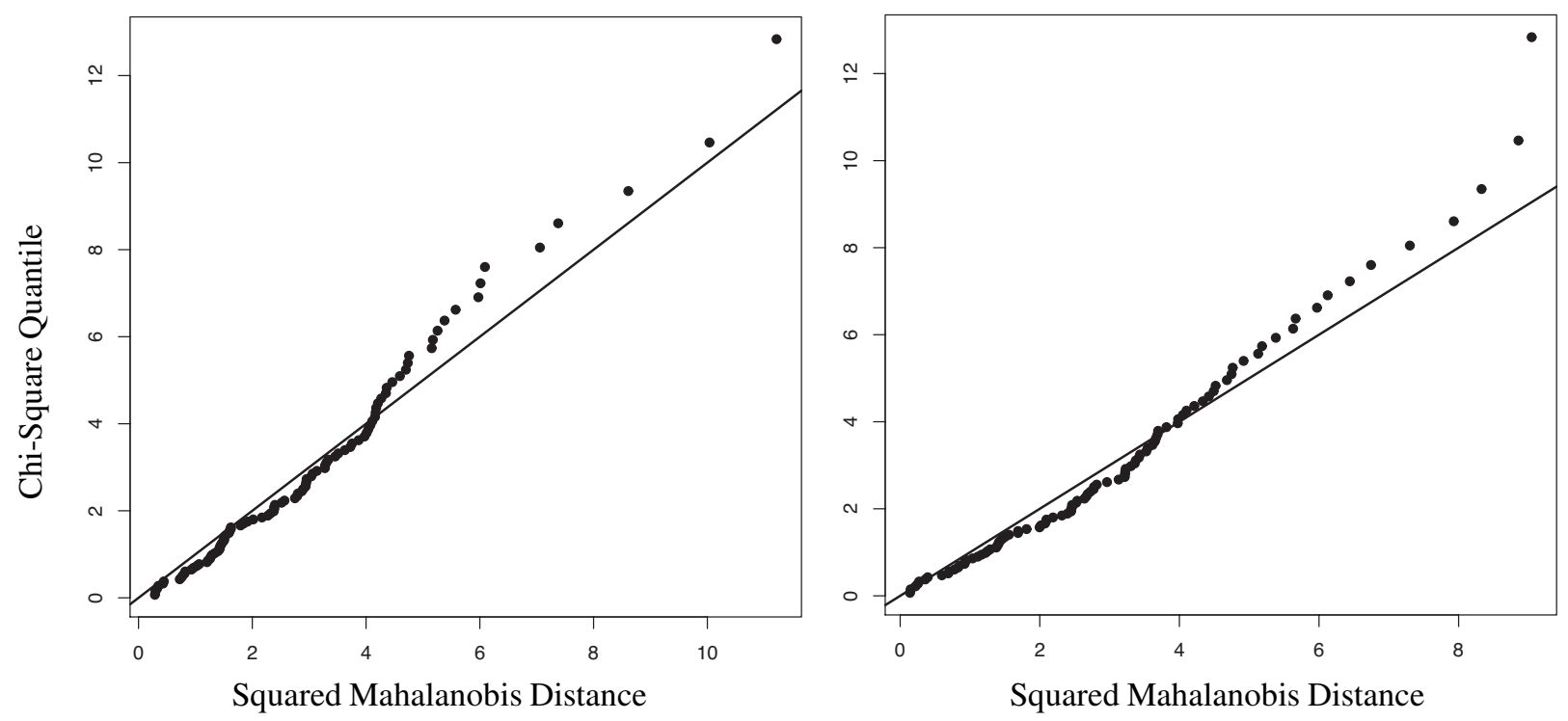

Figure 7. Q-Q plots corresponding to: (i) fourth set of undamaged data to test, using the first three principal components (PC1-PC3) in the actuator phase 4 (left) and (ii) damage 2 data, using the first three principal components (PC1-PC3) in the actuator phase 1 (right). The points of these Q-Q plots are close to the line $y=x$ thus indicating the multivariate normality of the data.

normal distribution with mean $\left(\begin{array}{ll}0 & 0\end{array}\right)^{T} \in \mathbb{R}^{2}$ and covariance matrix $\left(\begin{array}{cc}0.250 & 0.375 \\ 0.375 & 1.000\end{array}\right) \in$ $\mathcal{M}_{2 \times 2}(\mathbb{R})$. For bivariate normally distributed data, we expect to obtain a three-dimensional bell-shaped graph from the perspective plot. Similarly, in the contour plot, we can observe a similar pattern. Some examples of contour plots for the data we consider in this paper are given in Figures 9 and 10. These plots are similar to the contour plot of the bivariate normal distribution in Figure 8.

Finally, the univariate normality for each principal component and for each actuator phase is also tested. The results are presented in Table 2. As it can be observed, the univariate data is normally distributed in most of the cases. However, this do not imply multivariate normality.

\subsubsection{Testing a multivariate mean vector}

The objective of this paper is to determine whether the distribution of the multivariate random samples that are obtained from the structure to be diagnosed (undamaged or not) is connected to the distribution of the baseline. To this end, a test for the plausibility of a value for a normal population mean vector will be performed. Let $s \in \mathbb{N}$ be the number of principal components that will be considered jointly. We will also consider that: (a) the baseline projection is a multivariate random sample of a multivariate random variable following a multivariate normal distribution with known population mean vector $\boldsymbol{\mu}_{\mathrm{h}} \in \mathbb{R}^{s}$ and known variance-covariance 


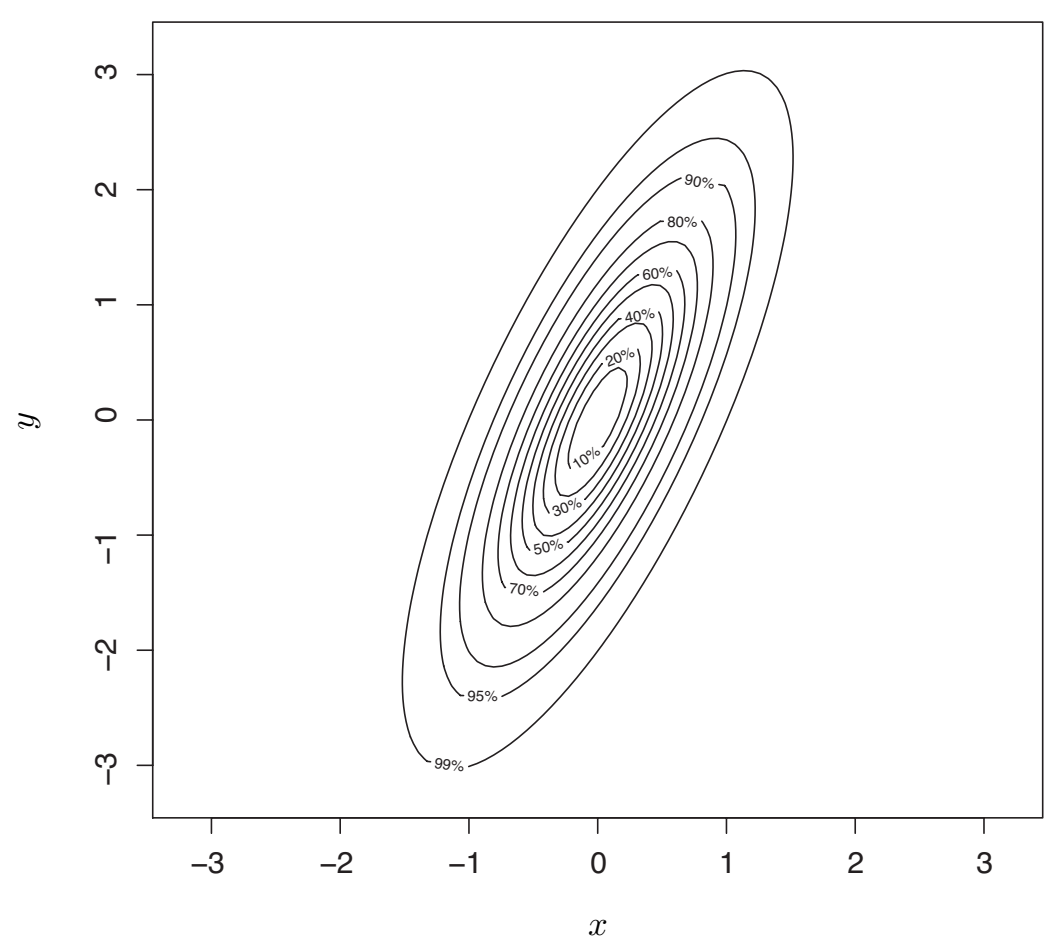

Figure 8. Contour plot for a bivariate normal distribution. The ellipses denote places of equal probability for the distribution and provide confidence regions with different probabilities.

matrix $\Sigma \in \mathcal{M}_{s \times s}(\mathbb{R})$; and (b) the multivariate random sample of the structure to be diagnosed also follows a multivariate normal distribution with unknown multivariate mean vector $\boldsymbol{\mu}_{\mathrm{c}} \in \mathbb{R}^{s}$ and known variance-covariance matrix $\Sigma \in \mathcal{M}_{s \times s}(\mathbb{R})$.

As said previously, the problem that we will consider is to determine whether a given $s$ dimensional vector $\boldsymbol{\mu}_{\mathrm{c}}$ is a plausible value for the mean of a multivariate normal distribution $N_{s}\left(\boldsymbol{\mu}_{\mathrm{h}}, \boldsymbol{\Sigma}\right)$. This statement leads immediately to a test of the hypothesis

$$
\begin{aligned}
& H_{0}: \boldsymbol{\mu}_{\mathrm{c}}=\boldsymbol{\mu}_{\mathrm{h}} \text { versus } \\
& H_{1}: \boldsymbol{\mu}_{\mathrm{c}} \neq \boldsymbol{\mu}_{\mathrm{h}},
\end{aligned}
$$

that is, the null hypothesis is 'the multivariate random sample of the structure to be diagnosed is distributed as the baseline projection' and the alternative hypothesis is 'the multivariate random sample of the structure to be diagnosed is not distributed as the baseline projection'. In other words, if the result of the test is that the null hypothesis is not rejected, the current structure is categorized as healthy. Otherwise, if the null hypothesis is rejected in favor of the alternative, this would indicate the presence of some structural changes in the structure.

The test is based on the statistic $T^{2}$-also called Hotelling's $T^{2}-$ and it is summarized below. When a multivariate random sample of size $\nu \in \mathbb{N}$ is taken from a multivariate normal 
Francesc Pozo, Ignacio Arruga, Luis E. Mujica, Elena Podivilova

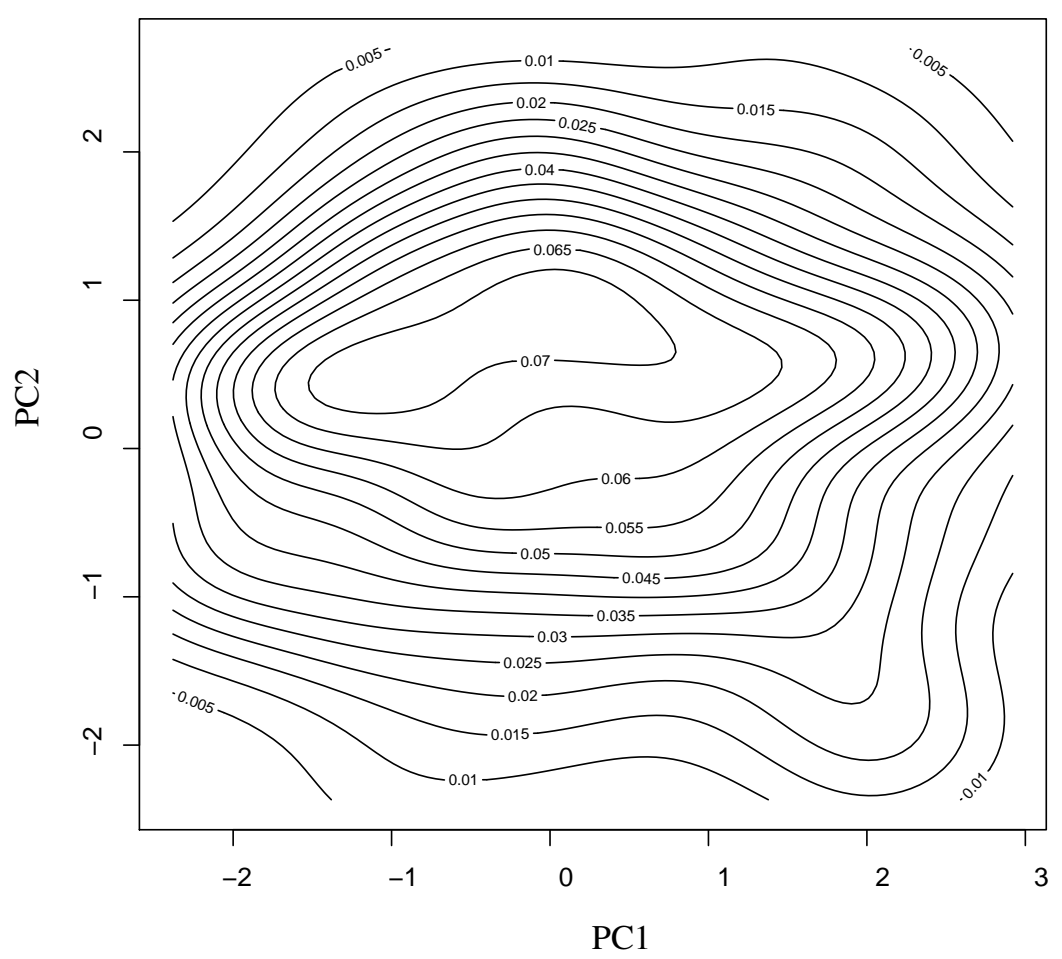

Figure 9. Contour plot for undamaged case (fourth set to test), PZT4 act., PC1-PC2. The contour lines are similar to ellipses of normal bivariate distribution from Fig. 8 that means that the distribution in this case is normal.

distribution $N_{s}\left(\boldsymbol{\mu}_{\mathrm{h}}, \boldsymbol{\Sigma}\right)$, the random variable

$$
T^{2}=\nu\left(\overline{\mathbf{X}}-\boldsymbol{\mu}_{\mathrm{h}}\right)^{T} \mathbf{S}^{-1}\left(\overline{\mathbf{X}}-\boldsymbol{\mu}_{\mathrm{h}}\right)
$$

is distributed as

$$
T^{2} \hookrightarrow \frac{(\nu-1) s}{\nu-s} F_{s, \nu-s}
$$

where $F_{s, \nu-s}$ denotes a random variable with an $F$-distribution with $s$ and $\nu-s$ degrees of freedom, $\overline{\mathbf{X}}$ is the sample vector mean as a multivariate random variable; and $\frac{1}{n} \mathbf{S} \in \mathcal{M}_{s \times s}(\mathbb{R})$ is the estimated covariance matrix of $\overline{\mathbf{X}}$.

At the $\alpha$ level of significance, we reject $H_{0}$ in favor of $H_{1}$ if the observed

$$
t_{\mathrm{obs}}^{2}=\nu\left(\overline{\mathbf{x}}-\boldsymbol{\mu}_{\mathrm{h}}\right)^{T} \mathbf{S}^{-1}\left(\overline{\mathbf{x}}-\boldsymbol{\mu}_{\mathrm{h}}\right)
$$




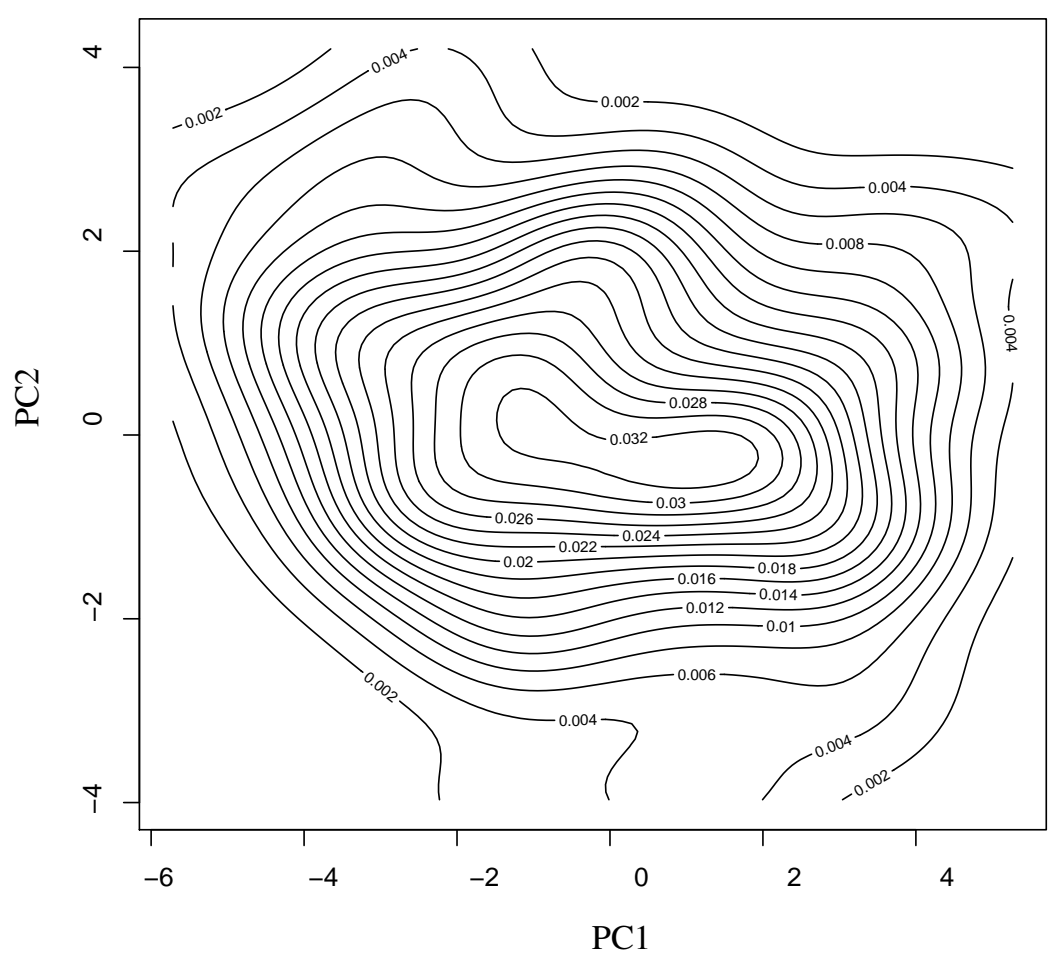

Figure 10. Contour plot for case D3, PZT1 act., PC1-PC2. The contour lines are similar to ellipses of normal bivariate distribution from Fig. 8 that means that the distribution in this case is normal.

is greater than $\frac{(\nu-1) s}{\nu-s} F_{s, \nu-s}(\alpha)$, where $F_{s, \nu-s}(\alpha)$ is the upper $(100 \alpha)$ th percentile of the $F_{s, \nu-s}$ distribution. In other words, the quantity $t_{\mathrm{obs}}^{2}$ is the damage indicator and the test is summarized as follows:

$$
\begin{aligned}
& t_{\mathrm{obs}}^{2} \leq \frac{(\nu-1) s}{\nu-s} F_{s, \nu-s}(\alpha) \Longrightarrow \text { Fail to reject } H_{0}, \\
& t_{\mathrm{obs}}^{2}>\frac{(\nu-1) s}{\nu-s} F_{s, \nu-s}(\alpha) \Longrightarrow \text { Reject } H_{0},
\end{aligned}
$$

where $F_{s, \nu-s}(\alpha)$ is such that

$$
\mathbb{P}\left(F_{s, \nu-s}>F_{s, \nu-s}(\alpha)\right)=\alpha
$$

where $\mathbb{P}$ is a probability measure. More precisely, we fail to reject the null hypothesis if $t_{\mathrm{obs}}^{2} \leq \frac{(\nu-1) s}{\nu-s} F_{s, \nu-s}(\alpha)$, thus indicating that no structural changes in the structure have been 
Table 2. Results of univariate normality tests when considering the first five principal components separately in the four actuator phases. "-" means lack of normality while "+" means normality.

\begin{tabular}{rcccc}
\hline & PZT1 act. & PZT2 act. & PZT3 act. & PZT4 act. \\
\hline Undamaged (baseline) & -+-++ & -++++ & -++++ & -++-+ \\
Undamaged (first set to test) & ---+- & -+++- & -++-+ & +-+++ \\
Undamaged (second set to test) & -++++ & -++++ & -++++ & -++-+ \\
Undamaged (third set to test) & --+++ & -++++ & -++++ & -++++ \\
Undamaged (fourth set to test) & -+-++ & -+++++ & -+++- & -++-+ \\
Undamaged (fifth set to test) & -+-++ & -++++ & -++++ & +++++ \\
D1 & -++++ & -++++ & -++-- & +++++ \\
D2 & -++++ & -++++ & -+++- & +++++ \\
D3 & +++++ & -++++ & -++++ & +++++ \\
D4 & -++++ & +++-+ & -++++ & -++++ \\
D5 & +++++ & -++++ & -+-++ & -++++ \\
\hline
\end{tabular}

found. Otherwise, the null hypothesis is rejected in favor of the alternative hypothesis if $t_{\mathrm{obs}}^{2}>\frac{(\nu-1) s}{\nu-s} F_{s, \nu-s}(\alpha)$, thus indicating the existence of some structural changes in the structure.

\section{EXPERIMENTAL RESULTS}

\subsection{Type I and Type II errors}

As said in Section 2.1 the experiments are performed in 4 independent phases: (i) piezoelectric transducer 1 (PZT1) is configured as actuator and the rest of PZTs as sensors; (ii) PZT2 as actuator; (iii) PZT3 as actuator; and (iv) PZT4 as actuator. In order to analyze the influence of each set of projections to the PCA model (score), the results of scores 1 to 3 (jointly), scores 1 to 5 (jointly) and scores 1 to 10 (jointly) have been considered. In this way, a total of 12 scenarios were examined. For each scenario, a total of 50 samples of 20 experiments each one ( 25 for the undamaged structure and 5 for the damaged structure with respect to each of the 5 different types of damages) plus the baseline are used to test for the plausibility of a value for a normal population mean vector, with a level of significance $\alpha=0.60$. Each set of 50 testing samples are categorized as follows: (i) number of samples from the healthy structure (undamaged sample) which were classified by the hypothesis test as 'healthy' (fail to reject $H_{0}$ ); (ii) undamaged sample classified by the test as 'damaged' (reject $H_{0}$ ); (iii) samples from the damaged structure 
(damaged sample) classified as 'healthy'; and (iv) damaged sample classified as 'damaged'. The results for the 12 different scenarios presented in Table 4 are organized according to the scheme in Table 3. It can be stressed from each scenario in Table 4 that the sum of the columns is constant: 25 samples in the first column (undamaged structure) and 25 more samples in the second column (damaged structure).

Table 3. Scheme for the presentation of the results in Table 4.

\begin{tabular}{ccc}
\hline & undamaged sample $\left(H_{0}\right)$ & damaged sample $\left(H_{1}\right)$ \\
\hline Fail to reject $H_{0}$ & Correct decision & Type II error (missing fault) \\
Reject $H_{0}$ & Type I error (false alarm) & Correct decision \\
\hline
\end{tabular}

In this table, it is worth noting that two kinds of misclassification are presented which are denoted as follows:

1. Type I error (or false positive), when the structure is healthy but the null hypothesis is rejected and therefore classified as damaged.

2. Type II error (or false negative), when the structure is damaged but the null hypothesis is not rejected and therefore classified as healthy. The probability of committing a type II error is called $\beta$.

It can be observed from Table 4 that Type I errors (false alarms) appear only when we consider scores 1 to 3 (jointly) and scores 1 to 5 (jointly), while in the last case (scores 1 to 10), all the decisions are correct.

\subsection{Sensitivity and specificity}

Two more statistical measures can be selected here to study the performance of the test: the sensitivity and the specificity. The sensitivity, also called as the power of the test, is defined, in the context of this work, as the proportion of samples from the damaged structure which are correctly identified as such. Thus, the sensitivity can be computed as $1-\beta$. The specificity of the test is defined, also in this context, as the proportion of samples from the undamaged structure that are correctly identified and can be expressed as $1-\alpha$.

The sensitivity and the specificity of the test with respect to the 50 samples in each scenario have been included in Table 6. For each scenario in this table, the results are organized as shown in Table 5.

It is worth noting that type I errors are frequently considered to be more serious than type II errors. However, in this application a type II error is related to a missing fault whereas a type I error is related to a false alarm. In consequence, type II errors should be minimized. Therefore a small level of significance of $1 \%, 5 \%$ or even $10 \%$ would lead to a reduced number 
Table 4. Categorization of the samples with respect to presence or absence of damage and the result of the test, for each of the four phases and considering the first score, the second score, scores 1 to 3 (jointly), scores 1 to 5 (jointly) and scores 1 to 10 (jointly).

\begin{tabular}{|c|c|c|c|c|c|c|c|c|}
\hline & \multicolumn{2}{|c|}{ PZT1 act. } & \multicolumn{2}{|c|}{ PZT2 act. } & \multicolumn{2}{|c|}{ PZT3 act. } & \multicolumn{2}{|c|}{ PZT4 act. } \\
\hline & $H_{0}$ & $H_{1}$ & $H_{0}$ & $H_{1}$ & $H_{0}$ & $H_{1}$ & $H_{0}$ & $H_{1}$ \\
\hline \multicolumn{9}{|l|}{ Score 1} \\
\hline Fail to reject $H_{0}$ & 22 & 13 & 21 & 7 & 18 & 13 & 22 & 12 \\
\hline Reject $H_{0}$ & 3 & 12 & 4 & 18 & 7 & 12 & 3 & 13 \\
\hline \multicolumn{9}{|l|}{ Score 2} \\
\hline Fail to reject $H_{0}$ & 21 & 2 & 24 & 18 & 18 & 5 & 22 & 14 \\
\hline Reject $H_{0}$ & 4 & 23 & 1 & 7 & 7 & 20 & 3 & 11 \\
\hline \multicolumn{9}{|l|}{ Scores 1 to 3} \\
\hline Fail to reject $H_{0}$ & 24 & 0 & 24 & 13 & 25 & 9 & 24 & 4 \\
\hline Reject $H_{0}$ & 1 & 25 & 1 & 12 & 0 & 16 & 1 & 21 \\
\hline \multicolumn{9}{|l|}{ Scores 1 to 5} \\
\hline Fail to reject $H_{0}$ & 21 & 0 & 23 & 0 & 21 & 0 & 20 & 0 \\
\hline Reject $H_{0}$ & 4 & 25 & 2 & 25 & 4 & 25 & 5 & 25 \\
\hline \multicolumn{9}{|l|}{ Scores 1 to 10} \\
\hline Fail to reject $H_{0}$ & 25 & 0 & 25 & 0 & 25 & 0 & 25 & 0 \\
\hline Reject $H_{0}$ & 0 & 25 & 0 & 25 & 0 & 25 & 0 & 25 \\
\hline
\end{tabular}

Table 5. Relationship between type I and type II errors.

\begin{tabular}{ccc}
\hline & undamaged sample $\left(H_{0}\right)$ & damaged sample $\left(H_{1}\right)$ \\
\hline Fail to reject $H_{0}$ & specificity $(1-\alpha)$ & false negative rate $(\beta)$ \\
Reject $H_{0}$ & false positive rate $(\alpha)$ & sensitivity $(1-\beta)$ \\
\hline
\end{tabular}

of false alarms but to a higher rate of missing faults. That is the reason of the choice of a level of significance of $60 \%$ in the hypothesis test.

The results show that the sensitivity of the test $1-\beta$ is close to $100 \%$, as desired, with an average value of $78 \%$. The sensitivity with respect to Score 1 to 5 and Score 1 to 10 is increased, 
Table 6. Sensitivity and specificity of the test for each scenario.

\begin{tabular}{|c|c|c|c|c|c|c|c|c|}
\hline & \multicolumn{2}{|c|}{ PZT1 act. } & \multicolumn{2}{|c|}{ PZT2 act. } & \multicolumn{2}{|c|}{ PZT3 act. } & \multicolumn{2}{|c|}{ PZT4 act. } \\
\hline & $H_{0}$ & $H_{1}$ & $H_{0}$ & $H_{1}$ & $H_{0}$ & $H_{1}$ & $H_{0}$ & $H_{1}$ \\
\hline \multicolumn{9}{|l|}{ Score 1} \\
\hline Fail to reject $H_{0}$ & 0.88 & 0.52 & 0.84 & 0.28 & 0.72 & 0.52 & 0.88 & 0.48 \\
\hline Reject $H_{0}$ & 0.12 & 0.48 & 0.16 & 0.72 & 0.28 & 0.48 & 0.12 & 0.52 \\
\hline \multicolumn{9}{|l|}{ Score 2} \\
\hline Fail to reject $H_{0}$ & 0.84 & 0.08 & 0.96 & 0.72 & 0.72 & 0.20 & 0.88 & 0.56 \\
\hline Reject $H_{0}$ & 0.16 & 0.92 & 0.04 & 0.28 & 0.28 & 0.80 & 0.12 & 0.44 \\
\hline \multicolumn{9}{|l|}{ Scores 1 to 3} \\
\hline Fail to reject $H_{0}$ & 0.96 & 0.00 & 0.96 & 0.52 & 1.00 & 0.36 & 0.96 & 0.16 \\
\hline Reject $H_{0}$ & 0.04 & 1.00 & 0.04 & 0.48 & 0.00 & 0.64 & 0.04 & 0.84 \\
\hline \multicolumn{9}{|l|}{ Scores 1 to 5} \\
\hline Fail to reject $H_{0}$ & 0.84 & 0.00 & 0.92 & 0.00 & 0.84 & 0.00 & 0.80 & 0.00 \\
\hline Reject $H_{0}$ & 0.16 & 1.00 & 0.08 & 1.00 & 0.16 & 1.00 & 0.20 & 1.00 \\
\hline \multicolumn{9}{|l|}{ Scores 1 to 10} \\
\hline Fail to reject $H_{0}$ & 1.00 & 0.00 & 1.00 & 0.00 & 1.00 & 0.00 & 1.00 & 0.00 \\
\hline Reject $H_{0}$ & 0.00 & 1.00 & 0.00 & 1.00 & 0.00 & 1.00 & 0.00 & 1.00 \\
\hline
\end{tabular}

in mean, to a $100 \%$. The average value of the specificity is $90 \%$.

\subsection{Reliability of the results}

The results in Table 8 are computed using the scheme in Table 7. This table is based on the Bayes' theorem [1], where $P\left(H_{1} \mid\right.$ accept $\left.H_{0}\right)$ is the proportion of samples from the damaged structure that have been incorrectly classified as healthy (true rate of false negatives) and $P\left(H_{0} \mid\right.$ accept $\left.H_{1}\right)$ is the proportion of samples from the undamaged structure that have been incorrectly classified as damaged (true rate of false positives).

\subsection{The receiver operating characteristic (ROC) curves}

An additional study has been developed based on the ROC curves to determine the overall accuracy of the proposed method. These curves represent the trade-off between the false pos- 
Table 7. Relationship between proportion of false negative and false positives.

\begin{tabular}{ccc}
\hline & undamaged sample $\left(H_{0}\right)$ & damaged sample $\left(H_{1}\right)$ \\
\hline Fail to reject $H_{0}$ & $P\left(H_{0} \mid\right.$ accept $\left.H_{0}\right)$ & true rate of false negatives \\
& true rate of false positives & $P\left(H_{1} \mid\right.$ accept $\left.H_{0}\right)$ \\
Reject $H_{0}$ & $P\left(H_{0} \mid\right.$ accept $\left.H_{1}\right)$ & $P\left(H_{1} \mid\right.$ accept $\left.H_{1}\right)$ \\
&
\end{tabular}

Table 8 . True rate of false positives and false negatives.

\begin{tabular}{|c|c|c|c|c|c|c|c|c|}
\hline & \multicolumn{2}{|c|}{ PZT1 act. } & \multicolumn{2}{|c|}{ PZT2 act. } & \multicolumn{2}{|c|}{ PZT3 act. } & \multicolumn{2}{|c|}{ PZT4 act. } \\
\hline & $H_{0}$ & $H_{1}$ & $H_{0}$ & $H_{1}$ & $H_{0}$ & $H_{1}$ & $H_{0}$ & $H_{1}$ \\
\hline \multicolumn{9}{|l|}{ Score 1} \\
\hline Fail to reject $H_{0}$ & 0.63 & 0.37 & 0.75 & 0.25 & 0.58 & 0.42 & 0.65 & 0.35 \\
\hline Reject $H_{0}$ & 0.20 & 0.80 & 0.18 & 0.82 & 0.37 & 0.63 & 0.19 & 0.81 \\
\hline \multicolumn{9}{|l|}{ Score 2} \\
\hline Fail to reject $H_{0}$ & 0.91 & 0.09 & 0.57 & 0.43 & 0.78 & 0.22 & 0.61 & 0.39 \\
\hline Reject $H_{0}$ & 0.15 & 0.85 & 0.13 & 0.88 & 0.26 & 0.74 & 0.21 & 0.79 \\
\hline \multicolumn{9}{|l|}{ Scores 1 to 3} \\
\hline Fail to reject $H_{0}$ & 1.00 & 0.00 & 0.65 & 0.35 & 0.74 & 0.26 & 0.86 & 0.14 \\
\hline Reject $H_{0}$ & 0.04 & 0.96 & 0.08 & 0.92 & 0.00 & 1.00 & 0.05 & 0.95 \\
\hline \multicolumn{9}{|l|}{ Scores 1 to 5} \\
\hline Fail to reject $H_{0}$ & 1.00 & 0.00 & 1.00 & 0.00 & 1.00 & 0.00 & 1.00 & 0.00 \\
\hline Reject $H_{0}$ & 0.14 & 0.86 & 0.07 & 0.93 & 0.14 & 0.86 & 0.17 & 0.83 \\
\hline \multicolumn{9}{|l|}{ Scores 1 to 10} \\
\hline Fail to reject $H_{0}$ & 1.00 & 0.00 & 1.00 & 0.00 & 1.00 & 0.00 & 1.00 & 0.00 \\
\hline Reject $H_{0}$ & 0.00 & 1.00 & 0.00 & 1.00 & 0.00 & 1.00 & 0.00 & 1.00 \\
\hline
\end{tabular}

itive rate and the sensitivity in Table 5 for different values of the level of significance that is used in the statistical hypothesis testing. Note that the false positive rate is defined as the complementary of the specificity, and therefore these curves can also be used to visualize the close relationship between specificity and sensitivity. It can also be remarked that the sensitivity is also called true positive rate or probability of detection [20]. More precisely, for each scenario 
and for a given level of significance the pair of numbers

$$
\text { (false positive rate, sensitivity) } \in[0,1] \times[0,1] \subset \mathbb{R}^{2}
$$

is plotted. We have considered 99 levels of significance within the range $[0.01,0.99]$ and with a difference of 0.01 . Therefore, for each scenario 99 connected points are depicted, as can be seen in Figures 11-13 when we consider scores 1 to 3 (jointly), scores 1 to 5 (jointly) and scores 1 to 10 (respectively).

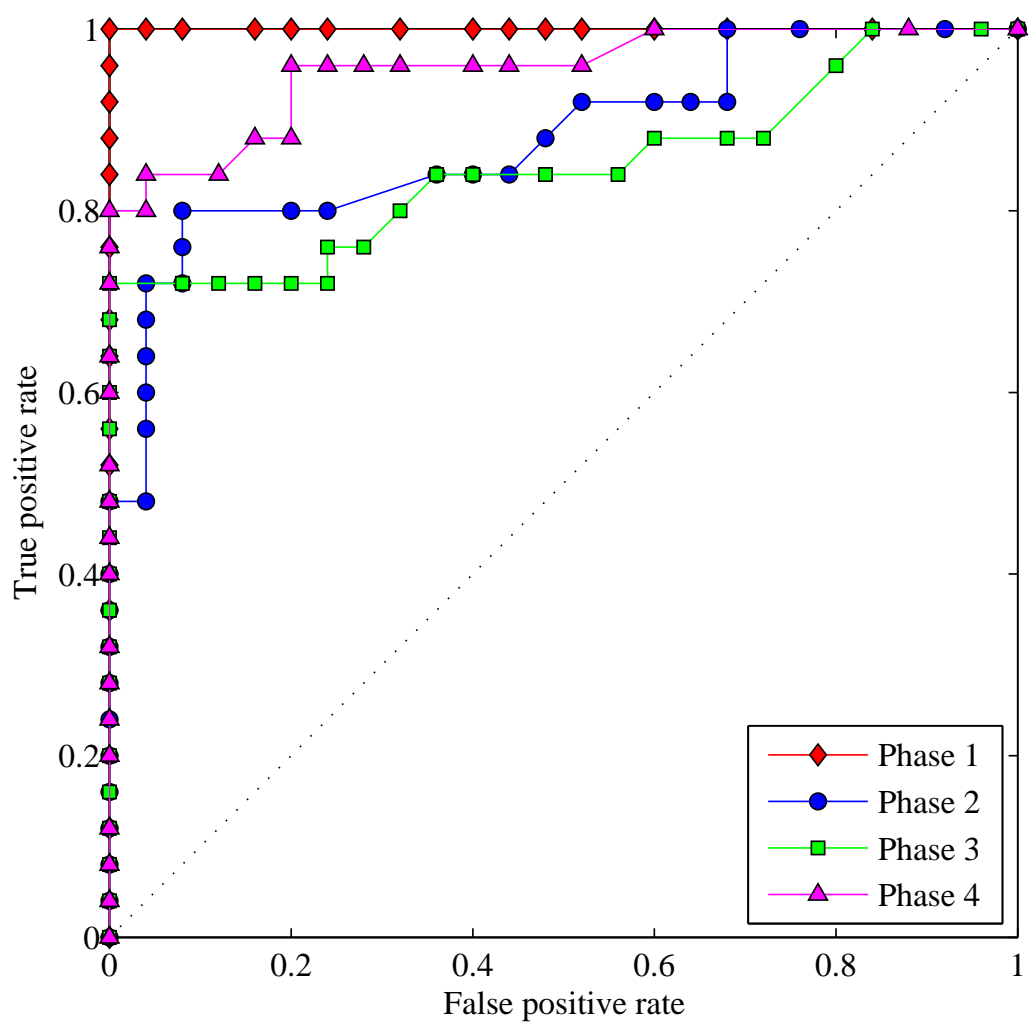

Figure 11. The receiver operating characteristic (ROC) curves for the scores 1 to 3 (jointly) in the four actuator phases.

The placement of these points can be interpreted as follows. Since we are interested in minimizing the number of false positives while we maximize the number of true positives, these points must be placed in the upper-left corner as much as possible. However, this is not always possible because there is also a relationship between the level of significance and the false positive rate. Therefore, a method can be considered acceptable if those points lie within the upper-left half-plane.

As said before, the ROC curves for the 12 possible scenarios are depicted in Figures 11-13. The best performance is achieved for the case of scores 1 to 3 in phase 1 (Figure 11) because 


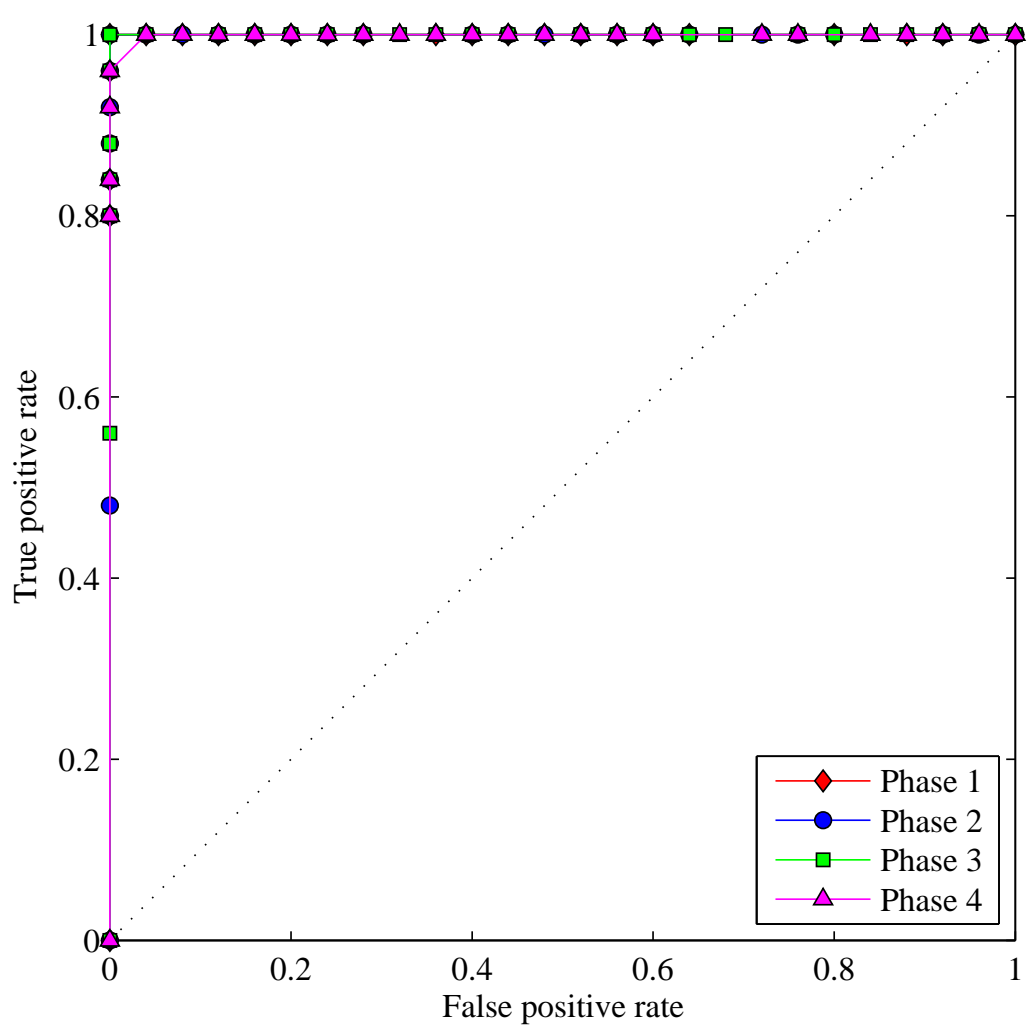

Figure 12. The receiver operating characteristic (ROC) curves for the scores 1 to 5 (jointly) in the four actuator phases.

all of the points are placed in the upper-left corner. In phases 2-4, the points lie in the upper left half-plain but not in the corner, which represents a very good behavior of the proposed method. When we consider the case of scores 1 to 5 (jointly) in Figure 12 and the case of scores 1 to 10 (jointly) in Figure 13 it can be observed that the area under the ROC curves is close to 1 in all of the actuator phases thus representing an excellent test.

Finally, we can say that the ROC curves provide a statistical assessment of the efficacy of a method and can be used to visualize and compare the performance of multiple scenarios.

\subsection{Analysis and discussion}

Multivariate tests allow to get better results in damage detection than univariate tests. This is perfectly illustrated in Figure 14 where a correct or wrong detections is represented as a function of the level of significance $\alpha$ used in the test. We can clearly characterize four different regions:

- $0<\alpha \leq 0.13$. In this region, all the five univariate tests and the multivariate statistical inference pass (correct decision). 


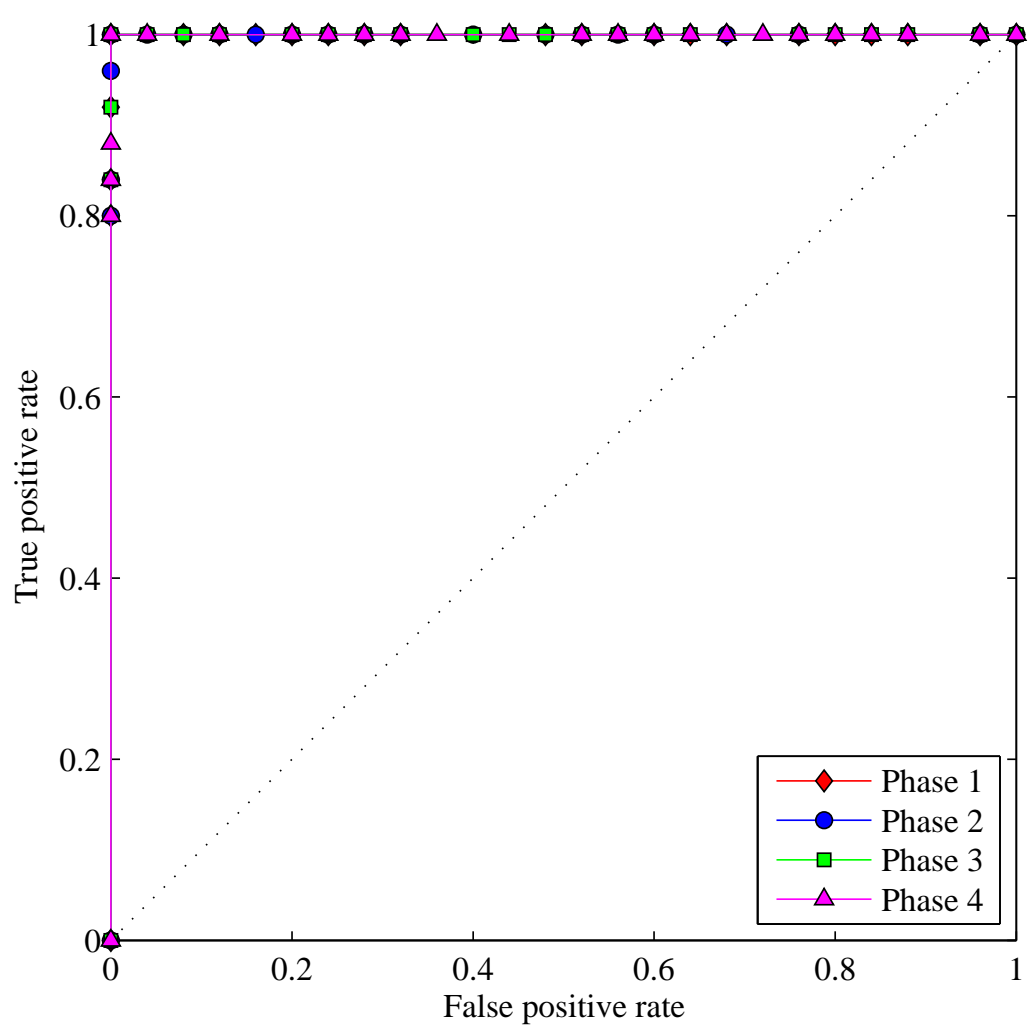

Figure 13. The receiver operating characteristic (ROC) curves for the scores 1 to 10 (jointly) in the four actuator phases.

- $0.13<\alpha \leq 0.62$. In this region, some of the five univariate tests fail (wrong decision) while the multivariate statistical inference pass (correct decision).

- $0.62<\alpha \leq 0.71$. In this region, all the five univariate tests fail (wrong decision) while the multivariate statistical inference pass (correct decision).

- $0.71<\alpha<1$. In this region, all the five univariate tests and the multivariate statistical inference fail (wrong decision).

It is worth noting that in the region $0.62<\alpha \leq 0.71$, that is, when the level of significance lies within the range $(0.62,0.71]$ the multivariate statistical inference using scores 1 to 5 (jointly) is able to offer a correct decision even though all of the univariate tests make a wrong decision.

The scenarios with the best results are those that considers scores 1 to 10 , because the false negative rate is $0 \%$ and the false positive rate is $0 \%$ for all the actuator phases. The results for scores 1 to 5 (jointly) are quite good, because the false negative rate is $0 \%$ for all actuators and the false positive rate is $7-17 \%$. 


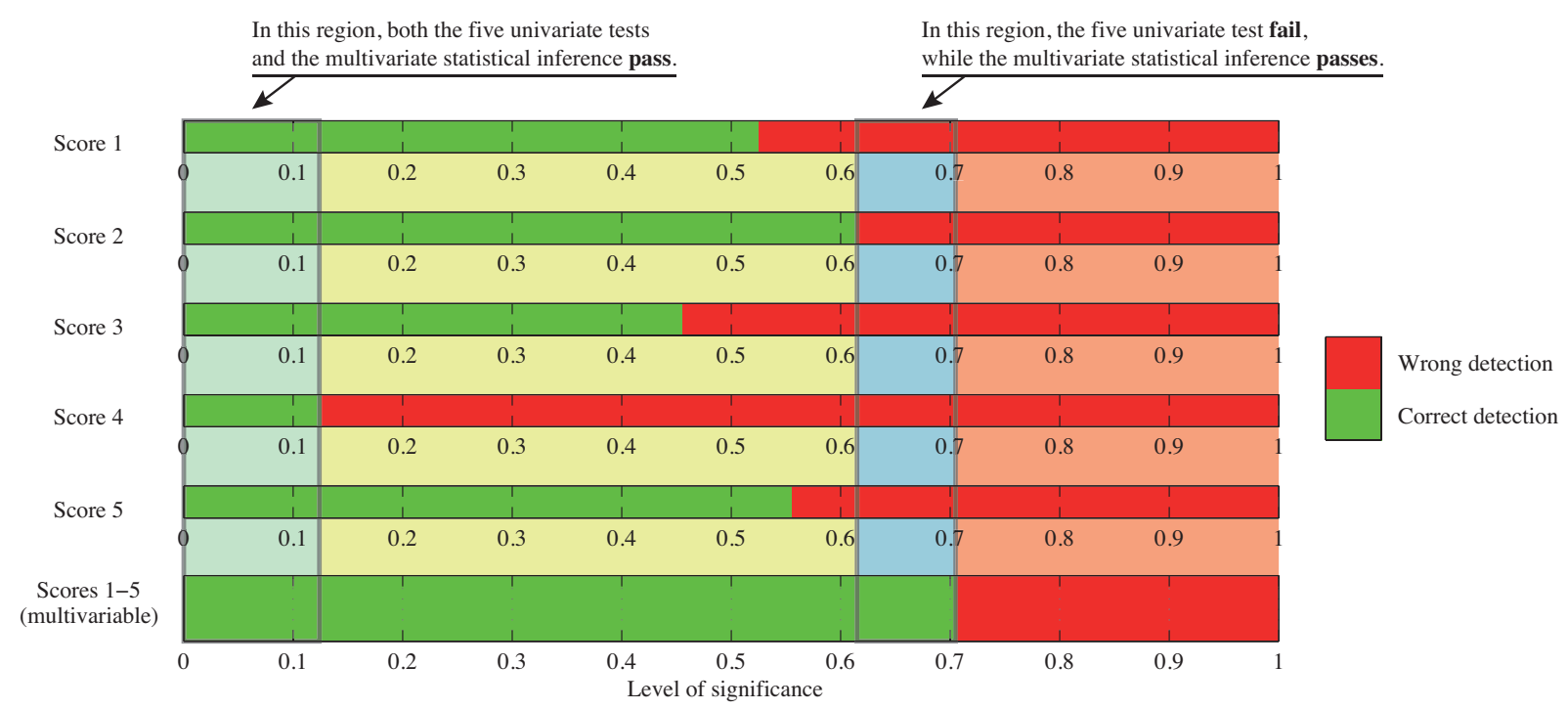

Figure 14. Multivariate test allow to get better results in damage detection that univariate tests. A correct or wrong detection is represented as a function of the level of significance where four regions can be identified.

\section{CONCLUDING REMARKS}

This paper has been focused on the development of a damage detection indicator that combines a data driven baseline model (reference pattern obtained from the healthy structure) based on principal component analysis (PCA) and multivariate hypothesis testing. A test for the plausibility of a value for a normal population mean vector has been performed. The results indicate that the test is able to accurately classify random samples as healthy or not.

\section{ACKNOWLEDGMENTS}

This work is supported by CICYT (Spanish Ministry of Economy and Competitiveness) through grant DPI2011-28033-C03-01.

\section{References}

[1] M.H. DeGroot and M.J. Schervish. Probability and Statistics. Pearson, 2012.

[2] C.R. Farrar, S.W. Doebling, and D.A. Nix. Vibration-based structural damage identification. Philosophical Transactions of the Royal Society A Mathematical Physical and Engineering Sciences, 359(1778):131-149, 2001.

[3] C.R Farrar and K. Worden. An introduction to structural health monitoring. Philosophical Transactions of the Royal Society - Series A: Mathematical, Physical and Engineering Sciences, 365(1851):303-315, 2007. 
[4] C.R Farrar and N.A.J Lieven. Damage prognosis: the future of structural health monitoring. Philosophical transactions. Series A, Mathematical, physical, and engineering sciences, 365(1851):623-32, February 2007.

[5] C.R. Farrar and K. Worden. Structural Health Monitoring: A Machine Learning Perspective. Wiley, 2012.

[6] S.P. Fassois and J.S. Sakellariou. Time-series methods for fault detection and identification in vibrating structures. Philosophical Transaction of the Royal Society A, 365:411-448, 2007.

[7] S.P. Fassois and J.S. Sakellariou. Statistical Time Series Methods for Structural Health Monitoring. In Christian Boller, Fou Kuo Chang, and Yozo Fujino, editors, Encyclopedia of Structural Health Monitoring, number ii, pages 443-472. John Wiley \& Sons, Ltd., 2009.

[8] N. Henze and B. Zirkler. A class of invariant consistent tests for multivariate normality. Communications in Statistics - Theory and Methods, 19(10):3595-3617, 1990.

[9] S. Korkmaz, D. Goksuluk and G. Zararsiz. MVN: An R Package for Assessing Multivariate Normality. MVN version 3.8, 2015.

[10] J. Kullaa. Sequential Structural Health Monitoring and Damage Detection. In Christian Boller, editor, Proceeding of Sixth European Workshop on Structural Health Monitoring, pages 1-8. DGZIP, 2012.

[11] J. Kullaa. Distinguishing between sensor fault, structural damage, and environmental or operational effects in structural health monitoring. Mechanical Systems and Signal Processing, 25(8):1-14, 2011.

[12] K.V. Mardia. Applications of some measures of multivariate skewness and kurtosis in testing normality and robustness studies. Sankhyā: The Indian Journal of Statistics, Series B (1960-2002), 36(2):115-128, 1974.

[13] L.E. Mujica, M. Ruiz, A. Güemes, and J. Rodellar. Contribution plots on PCA based indices for damage identification on structures. In 4th. ECCOMAS thematic conference on smart structures and materials, 2009.

[14] L.E. Mujica, J. Rodellar, A. Fernández, and A. Güemes. Q-statistic and T2-statistic PCA-based measures for damage assessment in structures. Structural Health Monitoring, 10(5):539-553, 2011.

[15] L.E. Mujica, M. Ruiz, F. Pozo, J. Rodellar, and A. Güemes. A structural damage detection indicator based on principal component analysis and statistical hypothesis testing. Smart Materials and Structures, 23(2):025014, 2014. 
[16] A.C. Rencher and W. F. Christensen. Methods of multivariate analysis. Wiley, 2012.

[17] P. Royston. Approximating the Shapiro-Wilk W test for non-normality. Statistics and Computing, 2(3):117-119, 1992.

[18] H. Sohn, K. Worden, and C.R. Farrar. Statistical Damage Classification Under Changing Environmental and Operational Conditions. Journal of Intelligent Material Systems and Structures, 13(9):561-574, 2002.

[19] H. Sohn, D.W. Allen, K. Worden, and C.R. Farrar. Statistical Damage Classification Using Sequential Probability Ratio Tests. Structural Health Monitoring, 2(1):57-74, March 2003.

[20] L. Yinghui and J.E. Michaels. Feature Extraction and Sensor Fusion for Ultrasonic Structural Health Monitoring Under Changing Environmental Conditions. IEEE Sensors Journals, 9(11):1462-1471, 2009.

[21] E. Zugasti, A. Gómez González, J. Anduaga, M.A. Arregui, and F. Martínez. NullSpace and AutoRegressive damage detection: a comparative study. Smarts Materials and Structures, 21:1-9, 2012. 